\title{
Nouveau type de prise d'eau en rivière
}

\section{A new type of river water intake}

\author{
PAR M. JOSÉ S. GANDOLFO \\ CHEF DU DÉPARTEMIENT D'HYURAULIOUE ET PROFESSEUR D'HYDRALLIOUE GÉNÉRALE

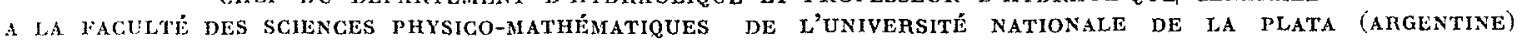

Fonctionnement hydraulique d'une prise d'eau. Nécessité de parer anx inconvénients dus all charriage des matériaux solides et à la formalion des dépots. Solations possibles.

Description da modèle. Définilion du matèriau de charriage. Condition's d'alimentation de la prise d'eau.

Elude $d u$ fonctionnement « normal 》 de la prise; fonctioninement en période d'etiage. Etude du forctionnement «anormal».

Possibilités et critères d'emploi de ce type de prise d'eau.

\begin{abstract}
Hydraulic aspects of water intakes. The need Li) guted against difficulties arising from trunsport and deposit of sediments. Possible solntions.

A description of the model. Grain size of sediment transported. Intake supply conditions.

Operation of the intake in normal conditions studied; operation at low stage flow. Operafion in abnormal conditions studied. Cases in which this type of water intake may be used. Criteria to be observed when using it.
\end{abstract}

\section{GENERALITES}

1. - L'établissement d'une prise d'eau en rivière n'est pas un problème exclusivement hydrodynamique. L'existence du transport solide en fait un aspect de l'hydraulique fluviale.

Ce genre d'ouvrages comprend un ensemble d'éléments qui, pendant leur fonctionnement hydraulique, réagissent les uns sur les autres; leur détermination peut se faire en considérant un ćcoulement à deux dimensions à travers chaque élément; c'est-à-dire en supposant une répartilion uniforme du débit. Dans la plupart des cas, cette méthode conduit à des projets d'ouvrages surdimensionnés.

En réalité le fonctionnement hydraulique de la prise d'eau considérée dans son ensemble s'écarte des conditions idéales correspondant aux hypothèses de calcul. En effet, on s'assure ainsi du passage des plus forts débits liquides: mais qu'il s'agisse de ce cas de fonctionnement ou de l'un quelconque de ceux que doit réaliser l'ouvrage, l'écoulement présente des caractéristiques particulières, résultant de l'ajustement tridimensionnel des filets liquides.

2. - Par ailleurs, le transport des matériaux solides par l'eau se présente sous deux aspects : a) Le transport en suspension; les modifications de concentration et de granulométrie du mélange de matériaux se produisent dans le dessableur, à l'aval de la prise d'eau;

b) Le transport par charriage, qui joue un rôle important dans le fonctionnement de la prise; en effet, la capacité de charriage de la rivière varie avec les débits liquides; de plus, il faut tenir compte des interférences entre la répartition du débit vers les divers éléments de la prise d'une part, et de la configuration des fonds dans son voisinage, d'autre part.

Nos maigres connaissances d'hydraulique fluviale concernant la capacité de charriage et l'évolution des fonds dans les cours d'eau ne peuvent guère nous aider à analyser les phénomènes locaux, compte tenu des perturbations que leur imposent les ouvrages.

3. - Les cas de fonctionnement sont nombreux et très divers; ils sont régis par l'ensemble suivant : apports de la rivière, barrage, prise d'eau. Aussi est-il impossible de prévoir pendant l'élaboration du projet les multiples cas qui peuvent se présenter, ainsi que le comportement de la rivière en présence des phénomènes qui 
se réalisent dans son lit une fois ce dernier modifié par la construction du barrage.

En ce qui concerne plus particulièrement les problèmes posés par la prise d'eau, problèmes qui dépassent les moyens d'analyse théorique de l'hydraulique fluviale, il pourront être résolus avec succès par une étude expérimentale sur modèle réduit.

L'aspect particulier de cette recherche ne doit pas faire perdre de vue son objectif fondamental, qui est la dérivation de l'eau; mais l'hydrodynamique permet de déterminer sans difficulté, quoique par excès, les dimensions des ouvrages. Par contre, le problème se complique en raison du transport par charriage de matériaux solides dont les dimensions couvrent l'ensemble de la courbe granulométrique correspondant à la rivière et qui, du fait de leurs caractéristiques ct des conditions d'écoulement dans le canal d'alimentation, ne peuvent être acheminés en totalité jusqu'au dessableur.

En conséquence, une partie au moins des matériaux charriés doit être éliminée dans les ouvrages de prise avant d'entrer dans la dérivation. Autrement, l'obstruction du premier tronçon de la dérivation et sa conséquence, l'insuffisant écoulement d'eau, posent, pour les ouvrages, un très grave problème.

4. - Il y a de nombreuses solutions possibles à cette question difficile; celles dont le résultat est très satisfaisant - mais elles sont rares -... sont :

a) Chambre de piégeage où s'accumulent momentanément les plus gros matériaux solides; lorsque cette chambre est comblée, on la vide, l'alimentation en eau de la dérivation étant alors presque toujours interrompue. La décharge brutale, à la rivière, des matériaux accumulés dans la chambre exige des conditions d'écoulement particulièrement efficaces, afin d'éviter le développement de dépôts susceptibles de compromettre le succès des nettoyages ultérieurs;

b) Décharges permanentes à travers le seuil de la prise, contrôlées ou non par des vannes, pour maintenir la hauteur des dépôts en dessous de la cote du seuil; ce type d'ouvrage convient notamment dans le cas des rivières qui charrient des matériaux de petites dimensions. Rappelons également, au sujet de l'écoulement dans ces décharges, qu'en temps de crue, l'augmentation des débits liquides et des concentrations s'accompagne d'une réduction progressive de la hauteur de chute sur l'ouvrage.

L'une et l'autre de ces solutions entraînent une forte dépense initiale; leur fonctionnement est compliqué et coûteux. Les frais d'entretien sont d'autant plus élevés que les organes mobiles sont plus nombreux et que la baisse d'efficacité hydraulique des ouvrages est plus sensible.

Du reste, la plupart des ouvrages de ce type résultent d'études expérimentales, et les solutions adoptées ne sont valables que dans les cas particuliers envisagés.

5. - Le but de la présente étude expérimentale est la mise au point de dispositions appor-

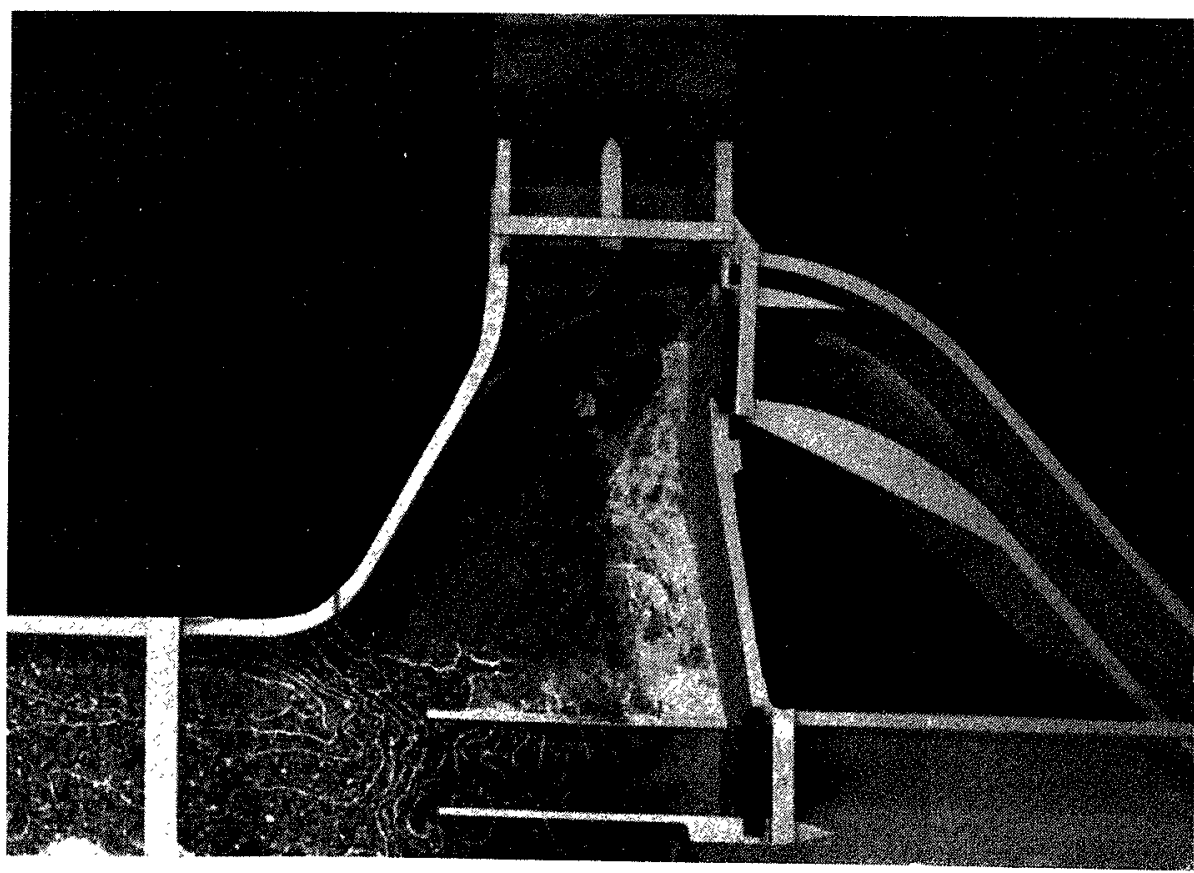

Pното 1. - Modèle de la première série 


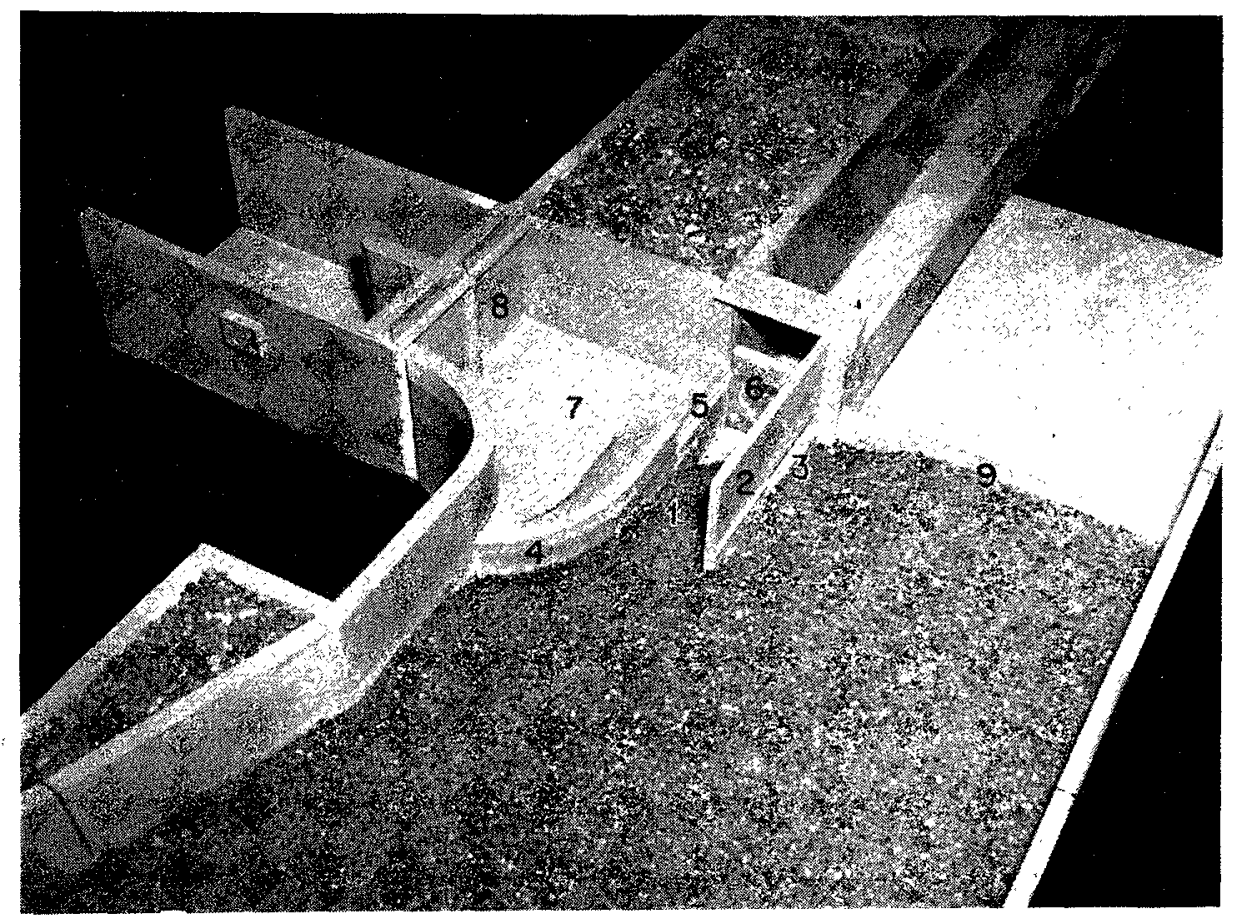

Phoто 2. - Modèle définitif du type de prise d'eau, quinzième série

1) Canal d'amenée. - 2) Mur guideau insubmersible. -- 3) Barbacane. - 4) Seuil de prise. - 5) Echancrure. - 6) Pertuis de chasse. - 7) Chambre de prise. 8) Vannes de la prise d'eau. - 9) Barrage.

tant une solution générale au problème en cause, c'est-à-dire permettant d'assurer un fonctionnemet correct de la prise dans les conditions les plus diverses.

Après avoir expérimenté quatorze types d'ouvrages, l'auteur parvint, avec le quinzième, aux dispositions satisfaisantes (brevetées) qui sont décrites ci-après.

\section{Description du modèle}

1. - Les essais ont été réalisés au Laboratoire d'Hydraulique «Guillermo C. Cespedes » à la Faculté des sciences physico-mathématiques de La Plata, en Argentine.

Le modèle a été fait à l'échelle du 1/40. Les forces de gravité sont prédominantes et l'écoulement est éminemment turbulent; en ce cas, les échelles sont réglées par la similitude de Reech-Froude. La distorsion inhérente aux modèles fluviaux de ce type est fixée compte lenu de la pente du lit, de la courbe granulométrique et du poids spécifique des matériaux charriés.

Les installations générales du modèle com- portent d'abord un groupe électro-pompe débitant dans un canal d'alimentation. A l'aval d'un tronçon de tranquillisation, le débit est mesuré par un déversoir Rehbock; de là, l'eau se déverse dans un bassin alimentant lui-même un autre canal qui conduit le débit, déjà mesuré, vers le modèle proprement dit. Des grilles de tranquillisation sont disposées dans ce dernier tronçon du canal d'alimentation; enfin, des guideaux assurent au débit pénétrant sur le modèle une répartition suffisamment uniforme et convenablement orientée selon l'axe de la rivière.

2. - En amont des ouvrages de prise et de dérivation, on dispose d'une section rectiligne de 100 mètres. La paroi du modèle opposée aux ouvrages de prise est constituée par une murette verticale suffisammient éloignée de ces ouvrages pour ne pas en perturber le fonctionnement. La rive, côté ouvrages de prise, est constituée de. gros graviers simulant un talus en enrochements.

On sait bien que le bon fonctionnement des ouvrages de dérivation dépend de leur heureuse. implantation dans la rivière. Les rives concaves se montrent favorables à l'étahlissement de 
prises fonctionnant sans incidents en toutes circonstances et dérivant des eaux relativement peu chargées en matériaux charriés.

Comme il a déjà été dit, le modèle représente une portion de rivière rectiligne, car il convenait d'éviter de placer les ouvrages étudiés dans des conditions de fonctionnement trop favorables; il n'y a évidemment aucune raison de se livrer à des études concernant une prise disposée sur une rive convexe; signalons cependant que le type ici décrit fonctionnerait beaucoup mieux que d'autres types employés couramment, en raison de la disposition des ouvrages qui le composent.

Il est certain que la prise ne pourra fonctionner dans les bonnes conditions exposées ci-après si son implantation est défectueuse. Plus le régime de la rivière sera torrentiel, plus il sera nécessaire d'implanter correctement la prise; cela favorise le fonctionnement de la prise pendant le plus longtemps possible et dans les conditions les plus propres à atténuer les répercussions des évolutions capricieuses du lit en présence des plus légères perturbations accidentelles. Les mêmes inconvénients peuvent surgir dans les cônes de déjection; mais, évidemment, dans le cas, bien hypothétique, du choix d'un tel emplacement pour la prise d'eau, il faut entreprendre dans le lit des travaux tendant ì stabiliser ce tronçon de la rivière.

3. - Sur le modèle, les ouvrages se composent du barrage, occupant la partie droite de la rivière, et des ouvrages de prise (photo 2). Ces derniers sont constitués essentiellement du canal d'amenée, du pertuis de chasse et des entrées; il n'est pas indispensable que celles-ci soient précédées d'une chambre de prise.

4. - Le canal d'amenée, dont l'axe est rectiligne et à peu près normal à celui du barrage, est délimité sur sa droite par un mur guideau insubmersible même pour les plus hautes caux; une longue ouverture ou «barbacane » est praliquée à sa base, près du barrage, afin de faciliter la concentration des eaux d'étiage vers la prise; comme on le verra plus loin, la présence de cette barbacane n'a aucune influence sur les autres cas de fonctionnement.

Sur sa gauche, le canal d'amenée est limité par un seuil de prise dont la partie amont, de forme convexe, se détache en saillie vers la rivière à partir du mur de protection de la berge, qui joue le rôle de mur de soutènement vis-à-vis du talus et assure une certaine étanchéité locale limitant les gradients d'infiltration vers les terres environnantes. Le seuil de prise peut être submergé en hautes caux.
5. - La quasi-totalité des malériaux charriés ayant pénétré dans le canal d'amenée est évacuée par les excédents de débit à travers le pertuis de chasse disposé à son extrémité aval. Certaines rivières ont un régime caractérisé par les crues régulières liées à la fonte des neiges, ce qui permet d'exploiter au maximum leurs débits habituels et conduit à réduire les excédents à des valeurs insignifiantes. Dans ce cas particulier, notre type de prise d'eau doit être soigneusement essayé sur un modèle réduit représentant un tronçon suffisamment long de la rivière en aval du barrage; là, il convient de s'assurer que le lit peut ètre maintenu, en présence d'un débit solide atteignant, ou presque, celui de la rivière en crue, les matériaux charriés étant entraînés sans former de dépôts ou de zones d'eaux mortes dans le voisinage de la partie aval des ouvrages; si de tels dépôts se formaient, cela nuirait à l'efficacité des chasses, et par suite, livrerait l'aceès de la prise aux matériaux charriés.

6. - Contre le pertuis de chasse, le seuil de prise présente une échancrure qui, comme on le verra, livre passage au débit dérivé.

Lorsque les débits à dériver sont importants, il convient de disposer une chambre de prise; on obtient ainsi une alimentation mieux répartie entre les différentes vannes de la prise; mais lorsque le débit à dériver est inférieur à $10 \mathrm{~m}^{3} / \mathrm{s}$ et que l'on se trouve dans des conditions favorables, l'entrée de la dérivation peut être reportée au niveau de l'échancrure.

7. - Au cours de son fonctionnement, le modèle est alimenté continuellement en matériaux charriés selon la concentration et la granulométrie nécessitées par chaque essai. Le charriage en suspension n'est pas pris en considération, étant donné qu'il est étranger à l'objet de la recherche; c'est d'ailleurs au dessableur qu'incombe Ia mission de modifier la concentration et la granulométrie du débit solide dérivé en suspension.

Les matériaux charriés, entrainés vers le barrage, le pertuis de chasse et la prise, sont recueillis dans des bacs séparés; ils sont triés et pesés afin d'établir leur granulométrie respective. De même, on détermine le poids de matériau de charriage utilisé dans chaque essai, et la granulométrie correspondante est relevée sur un échantillon.

Il ne faut pas s'attendre à une correspondance rigoureuse entre les matériaux classés alimentant le modèle et l'ensemble de ceux qui sont recueillis dans les bacs an terme de leur passage dans les divers éléments des ouvrages. D'une part, en raison même du mécanisme discontinu du 
transport par charriage caractérisé par la saltation, un tri s'opère dans l'ensemble du lit; d'autre part, des tris se produisent localement par suite des perturbations apportées à l'écoulement par des obstacles fixes dans la rivière. Enfin, la partic la plus fine du matériau est partiellement mise en suspension dans les zones les plus turbulentes; c'est seulement dans le bac collecteur de la dérivation que des précautions particulières doivent être prises afin de pouvoir mesurer la quantité totale de matériaux dérivés avec le débil liquide.

8. - Les niveaux sont mesurés lors de chaque essai. Dans la moitié environ des essais, les cotes du fond sont fixées, dans l'axe du barrage (sur le modèle) et dans l'axe du canal d'amenée. Dans chaque cas, les lignes de niveau des dépôts du lit sont tracées à la fin de l'essai : elles figurent les conditions permanentes, ou de régime, admises comme établies au début de chaque expérience; c'est-à-dire que, pendant la durée de l'expérience destinée à relever telle ou telle incidence particulière, il ne se produit que de faibles modifications dans la disposition des fonds qui restent presque identiques à ceux définis par les lignes de niveau.

Signalons que la configuration générale des dépòts changerait partiellement au cas où, soucieux de tenir compte des matériaux en suspension apportés par les crues, on effectuerait les expériences avec des eaux chargées. Mais ces modifications, dues à la décantation des matériaux fins, intéressent uniquement des zones d'eaux relativement calmes; il ne s'agit évidemment ni du canal d'amenée au voisinage de l'entrée, ni du chenal principal dans lequel se produisent les mouvements du fond les plus importants vis-à-vis du comportement du nouveau type de prise.

\section{Le matériau de charriage}

1. - Les essais sont conduits avec des matériaux caractérisés par des courbes granulométriques formant des quarts de cercle en représentation cartésienne. Les matériaux charriés dans la nature ont tendance à se présenter sous celte forme, lorsque les échantillons analysís ne proviennent pas de zones où s'effectue un triage naturel; dans ce cas, on mélange plusieurs échantillons prélevés en divers points d'une même section transversale.

Afin de vérifier la souplesse du fonctionnement et les larges possibilités d'utilisation du type de prise d'eau préconisé, les recherches faites onl portí sur des matériaux de charriage caractérisés sensiblement par les granulométries suivantes :
Granulométrie des matériaux de charriagl:

\begin{tabular}{|c|c|c|c|c|}
\hline $\begin{array}{c}\text { Diamètre } \\
\text { min }\end{array}$ & $\begin{array}{c}\text { Matériau } \\
n^{\circ} 1\end{array}$ & $\begin{array}{c}\text { Matériau } \\
10^{\circ} 3\end{array}$ & $\begin{array}{c}\text { Matériau } \\
n^{\circ} 6\end{array}$ & $\begin{array}{c}\text { Matériau } \\
n^{\circ} 8\end{array}$ \\
\hline 25,4 & & & & \\
\hline 19,5 & & & & \\
\hline 15,875 & & & & \\
\hline 12,7 & & & & \\
\hline 9,525 & $\begin{array}{r}0,0 \\
11,9\end{array}$ & 11,7 & & \\
\hline $\begin{array}{l}0,50 \\
4,69\end{array}$ & 7,9 & 8,7 & 3,4 & \\
\hline 3,32 & 8,5 & 10,8 & 8,7 & \\
\hline 2,362 & 7,4 & 7,6 & 10,0 & 3,8 \\
\hline 1,65 & 6,8 & 9,0 & 10,4 & 9,4 \\
\hline 1,16 & 5,6 & 5,9 & 9,5 & 10,5 \\
\hline 0,503 & 9,7 & 12,2 & 19,1 & 23,5 \\
\hline 0,246 & & & & \\
\hline
\end{tabular}

Le poids spécifique de ces matériaux est environ $2,6 \mathrm{~g} / \mathrm{cm}^{3}$. On note, pour chaque essai, la concentration en matériaux charriés réalisée; cette concentration est de l'ordre de 0,5 à $1 \%$

2. - Avant de procéder aux observalions nécessaires, le modèle est réglé jusqu’à ce qué les dépôts dans la rivière et au voisinage des ouvrages aient évolué et atteint leur configuralion de régime; en même temps on corrige les débits au barrage, au pertuis de chasse et à la prise, compte tenu de la répartition recherchíc et jusqu'à obtenir un réglage correct de l'écoulement.

Pour que le fonctionnement du canal d'amenéc soit correct, le mécanisme des mouvements du fond doit y être parvenu au terme de son évolution; le régime d'équilibre de l'écoulement dans cette zone dépend du niveau du plan d'eau et des débits liquide et solide qui le parcourent.

\section{Uniformité d'alimentation de la prise d'eau}

1. - Le caractère capricieux de l'écoulement de nombreuses rivières - notamment celles qui multiplient leurs ramifications sinueuses parmi de vastes banes alluviaux - pose, en outre, des problemes tout à fait particuliers en ce qui 
concerne l'exploitation des prises d'eau, en raison de la diversité des conditions dans lesquelles celles-ci peuvent être alimentées. Ces difficultés se font tout particulièrement sentir dans le cas des barrages fixes, où il est impossible d'agir sur le tracé du chenal principal dans le troncon de la rivière immédiatement en amont. Mais on ne doit pas s'attendre à de bien meilleures conditions d'alimentation dans le cas des barrages mobiles, car s'il est vrai que le contrôle de l'écoulement est alors plus souple au voisinage des ouvrages, par contre, l'exhaussement des dépôts alluviaux consécutifs à la surélévation du plan d'eau, la formation d'îlots couverts de végétation, et le tassement de certains bancs ne permettent pas d'imposer au chenal principal le tracé le plus favorable; on peut, certes, y parvenir, mais au prix d'une grande perte d'eau correspondant au débit qu'on doit laisser s'écouler librement pendant assez longtemps en renonçant à le diriger vers les ouvrages d'exploitation.

Tant au cours de l'exploitation des ouvrages réels qu'au cours des études de prises d'eau sur modèle réduit, on a pu apprécier le caractère de ces difficultés. Il est fort difficile de définir, par voie expérimentale, les moyens propres à assurer les meilleures conditions de prise d'eau tout au long des multiples cas de fonctionnement pouvant découler aussi bien des ouvrages euxmêmes que de leurs répercussions sur le comportement des fonds dans leur voisinage.

2. - Le type de prise d'eau décrit présente, dans les conditions normales, un seul mode de fonctionnement au cours des crues. En effet, ce mode de fonctionnement n'est influencé ni par les débits liquides ni par la concentration nu la granulométrie caractérisant le débit de charriage; il ne sera pas modifié non plus par le débit empruntant le canal d'amenée ou par la répartition de ce débit entre la prise et le pertuis de chasse. L'unique précaution à prendre est de lever suffisamment les vannes de chasse, pour éviter l'arrêt des plus gros matériaux susceptibles de se présenter dans le tronçon de rivière où sont implantés les ouvrages; il n'y a pas lieı de tenir compte des cailloux plus gros pouvant survenir occasionnellement.

En effet, on disposera toujours d'un chenal permanent alimentant directement le canal d'amenée avec un débit largement suffisant. C'est là qu'intervient l'effet d'entonnement de l'entrée du canal d'amenée. Le mur suideau insubmersible répartit les débits entre le barrage et les ouvrages de prise; à son extrémité amont, le materiau de fond est l'objet d'un brassage intense.

La partie courbe du seuil de prise provoque un affouillement local. Pour une mème hauteur d'eau, l'importance de cet affouillement est d'autant plus grande que le débit passant par le canal d'amenée est plus élevé et que la concentration des eaux de la rivière en matériaux charriés est plus faible. Si l'on compare le comportement de plusieurs rivières présentant les mêmes caractéristiques hydrauliques et ẻquipẻes des mêmes ouvrages, on constate que les érosions locales augmentent au fur et à mesure que les matériaux charriés ont une granulométrie plus fine.

L'érosion due à la partie courbe du seuil de prise s'accompagne d'un mouvement hélicoïdal qui, presque toujours, tend à éviter le dépôt contre le parement vertical de l'ouvrage, des matériaux de charriage entraînés dans cette zone.

Le mouvement hélicoïdal provoque un triage et une stratification des matériaux solides, les plus gros étant vers le fond. D’une façon générale, les matériaux qui arrivent dans cette zone d'érosion parcourent ensuite les dépôts formés dans le canal d'amenée (photo 3 ). Dans certains cas, lorsque le matériau charrié est très fin, l'affouillement près du seuil est très important et il peut s'étendre jusqu'à rejoindre celui de l'extrémité amont du mur guideau; dans ces conditions, une part importante des matériaux atteignant la zone considérée est déviée vers le barrage (photo 4 ).

Par l'action combinée du mur guideau et de la courbure du seuil de prise, les dépôts s'abaissent à l'entrée et à l'intérieur du canal d'amenée, la section de l'écoulement demeurant ainsi très grande.

Aussi pourra-t-on facilement, quel que soit le niveau du plan d'eau, diriger sur le canal d'amence d'importantes fractions du débit de la rivière.

3. - La configuration générale de la rivière au voisinage de la prise change selon l'importance des débits liquides et solides linéaires (par unité de largeur).

Au cours des grandes crues, étant donné la valeur ćlevée du débit linéaire au barrage, les dépôts présentent un aspect assez uniforme et ont tendance à orienter le chenal principal vers la zone du barrage (photos 5 et 6 ). Dans ce cas, tous les éléments des ouvrages sont abondamment alimentés.

A la décrue, à mesure que les débits diminuent, le chenal dirigé vers le canal d'amenée prend une importance croissante; cela provient de la permanence $d u$ débit s'écoulant dans ce canal vers le pertuis de chasse et la prise. En conséquence, par suite de la diminution des débits linéaires au barrage et de l'augmentation 


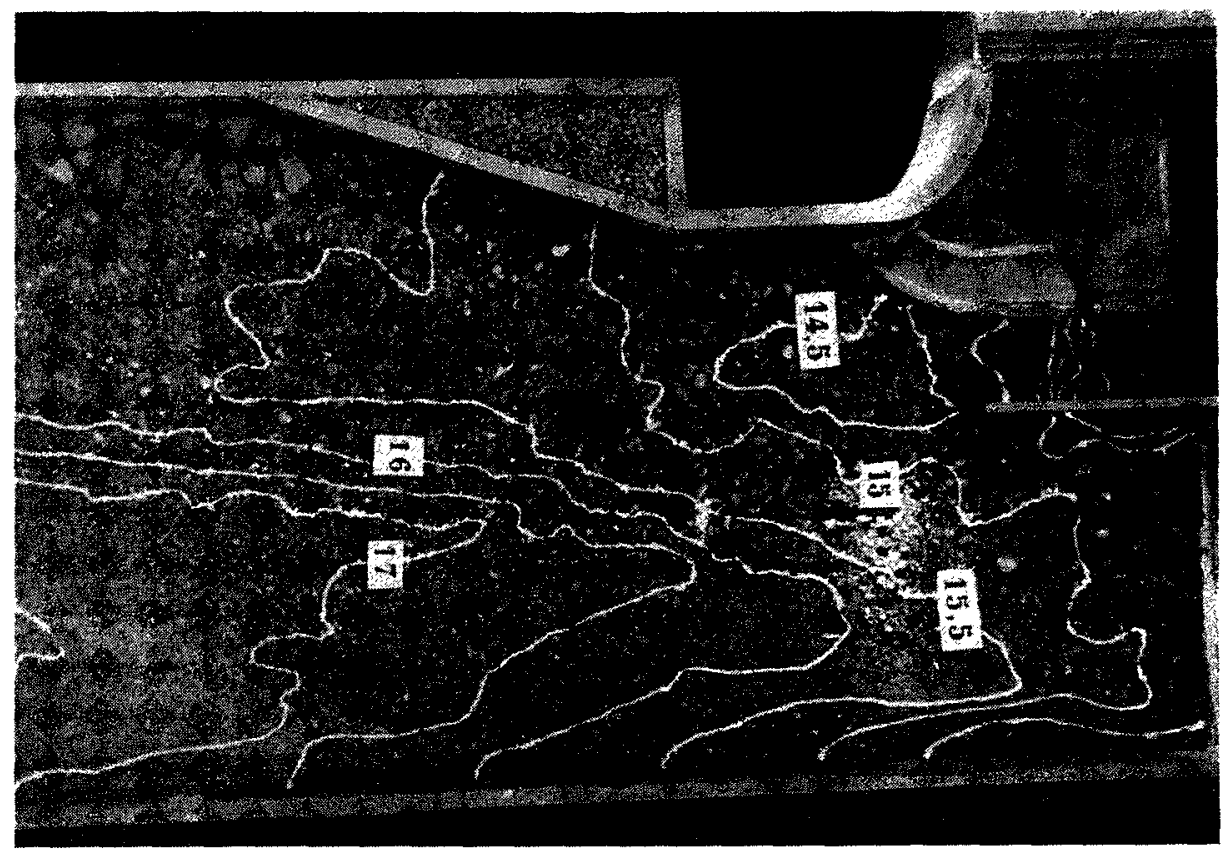

Pното 3. - Expérience 25. Lignes de niveau en fonctionnement normal. Répartition du débit liquide :

barrage : $42,9 \%$ — chasse : $31,7 \%$ - prise d'ean : $25,4 \%$

Matériau solide de charriage $n^{\circ} 1$

Concentration dans la rivière en amont de la prise d'eau : 1,040 \%

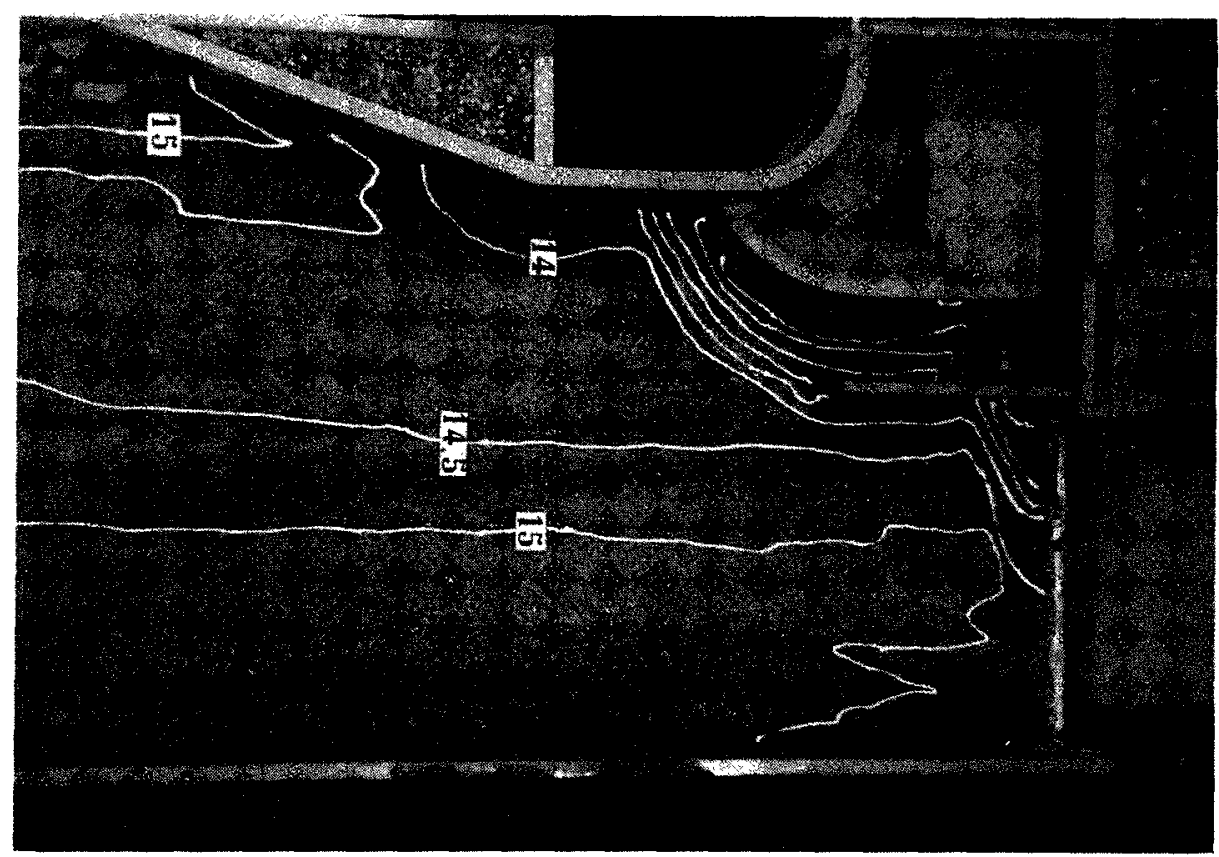

PHoто 4. - Expérience 32. Lignes de niveau en fonctionnement normal Répartition du débit liquide :

barrage : $\$ 2,9 \%$ - chasse : $31,7 \%$ - prise d'eau : $25,4 \%$

Matériau solide de charriage $n^{\circ} 8$

Concentration dans la rivière en amont de la prise d'eau : 0,472\%o 


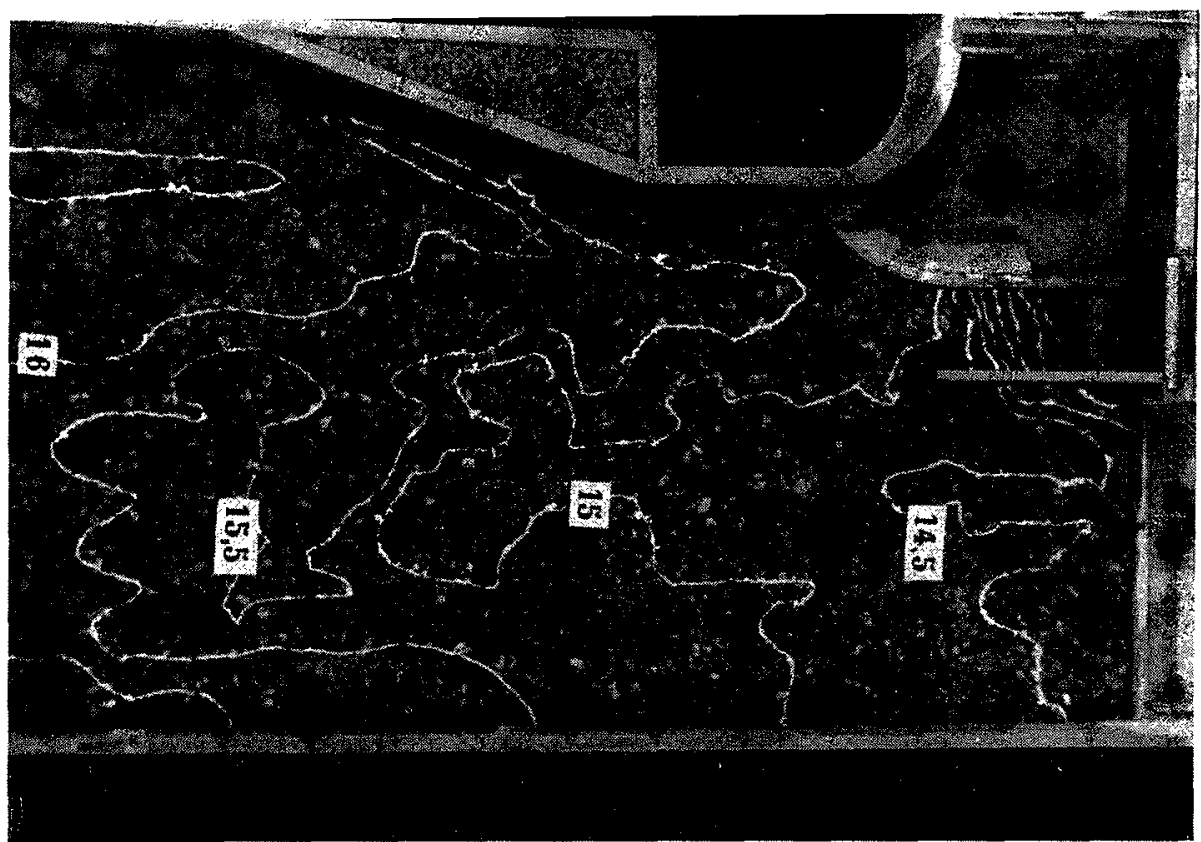

Photo 5. - Expérience 21. Lignes de niveau en fonctionnement normal Répartition du débit liquide :

barrage : $79 \%$ - chasse : $11,7 \%$ - prise d'eau : $9,3 \%$.

Matériau solide de charriage $n^{\circ} 1$

Concentration dans Ja rivière en amont de la prise d'eau : $0,987 \%$

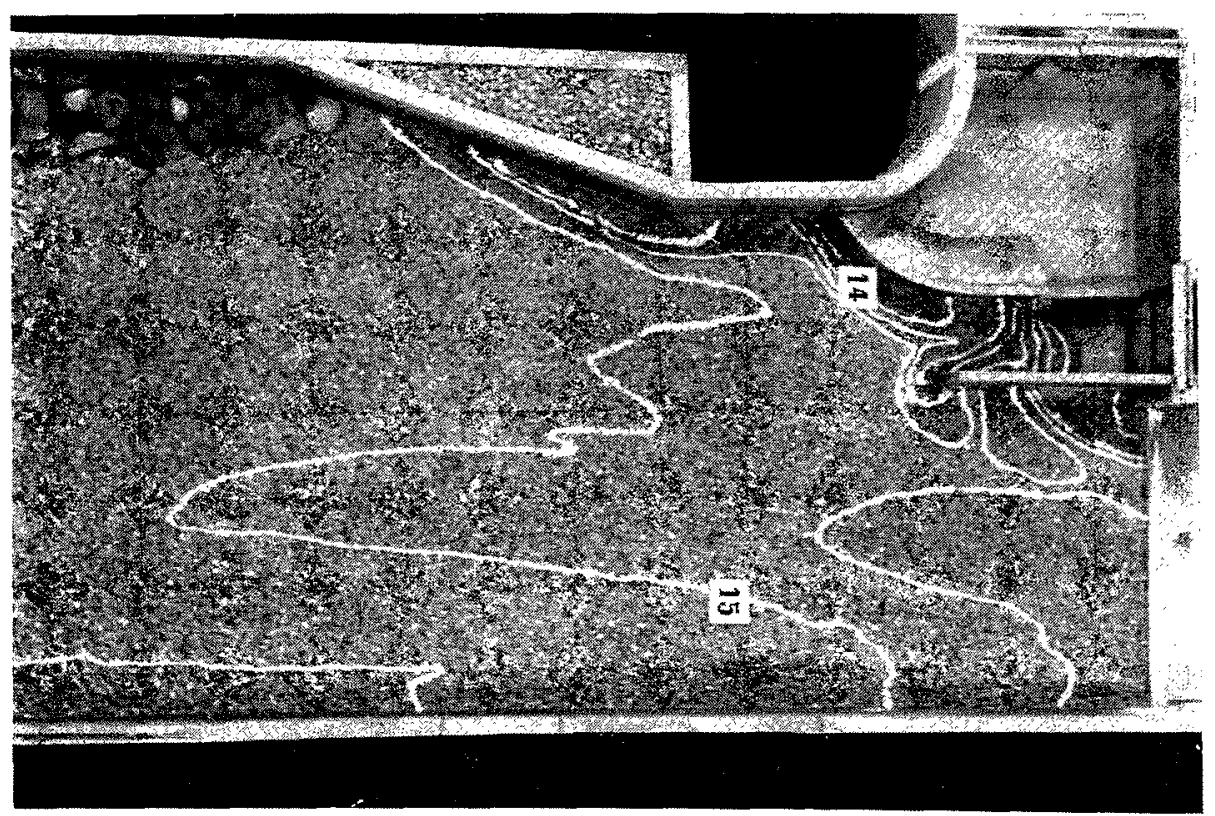

Рното 6. - Expérience 11. Lignes de niveau en fonctionnement normal Répartition du débit liquide :

barrage: $79 \%$ - chasse : $11,7 \%$ - prise d'eau : $9,3 \%$

Matériau solide de charriage $n^{\circ} 6$

Concentration dans la rivière en amont de la prise d'eau : $0,434 \%$ 


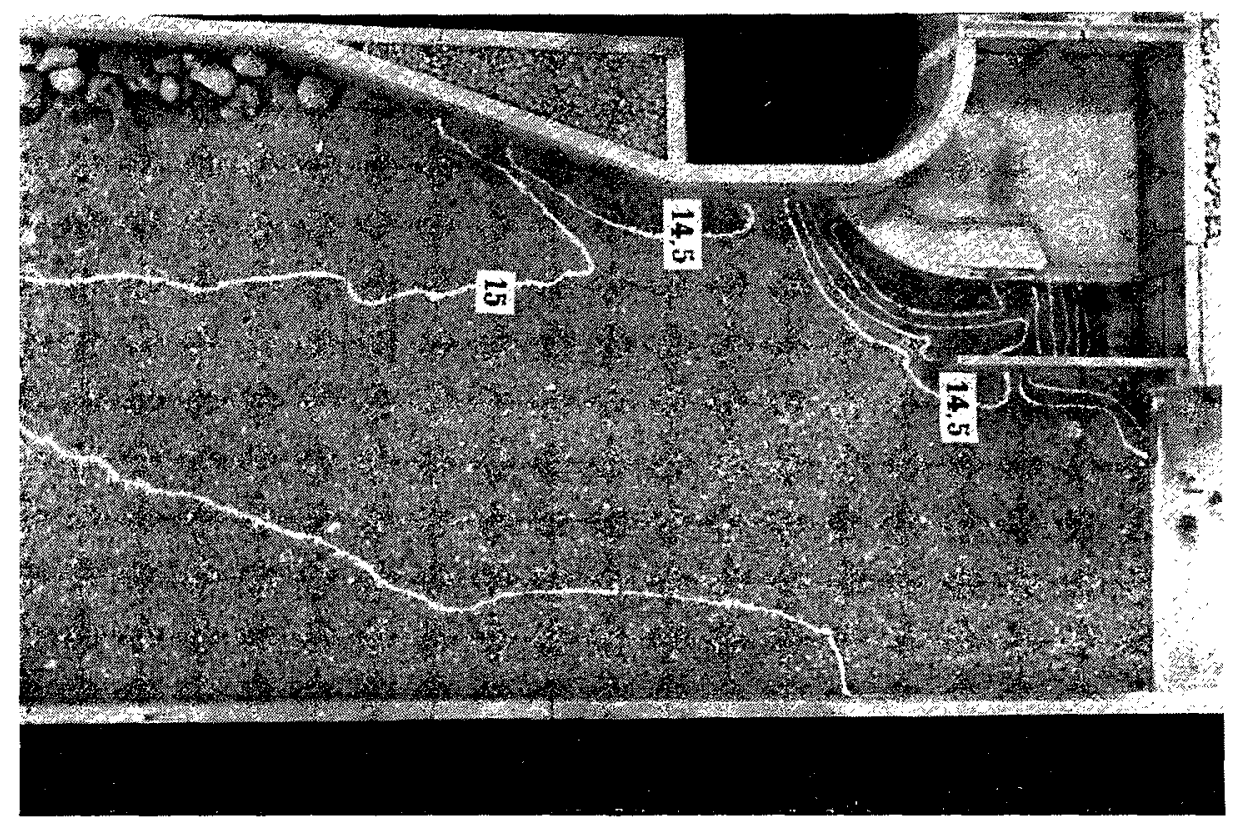

Pното 7. - Expérience 16. Lignes de niveau en fonctionnement normal Répartition du débit liquide :

barrage : $65,2 \%$ — chasse : $19,3 \%$ - prise d'eau : $15,5 \%$

Matériau solide de charriage $n^{\circ} 6$

Concentration dans la rivière en amont de la prise d'eau : $0,867 \%$

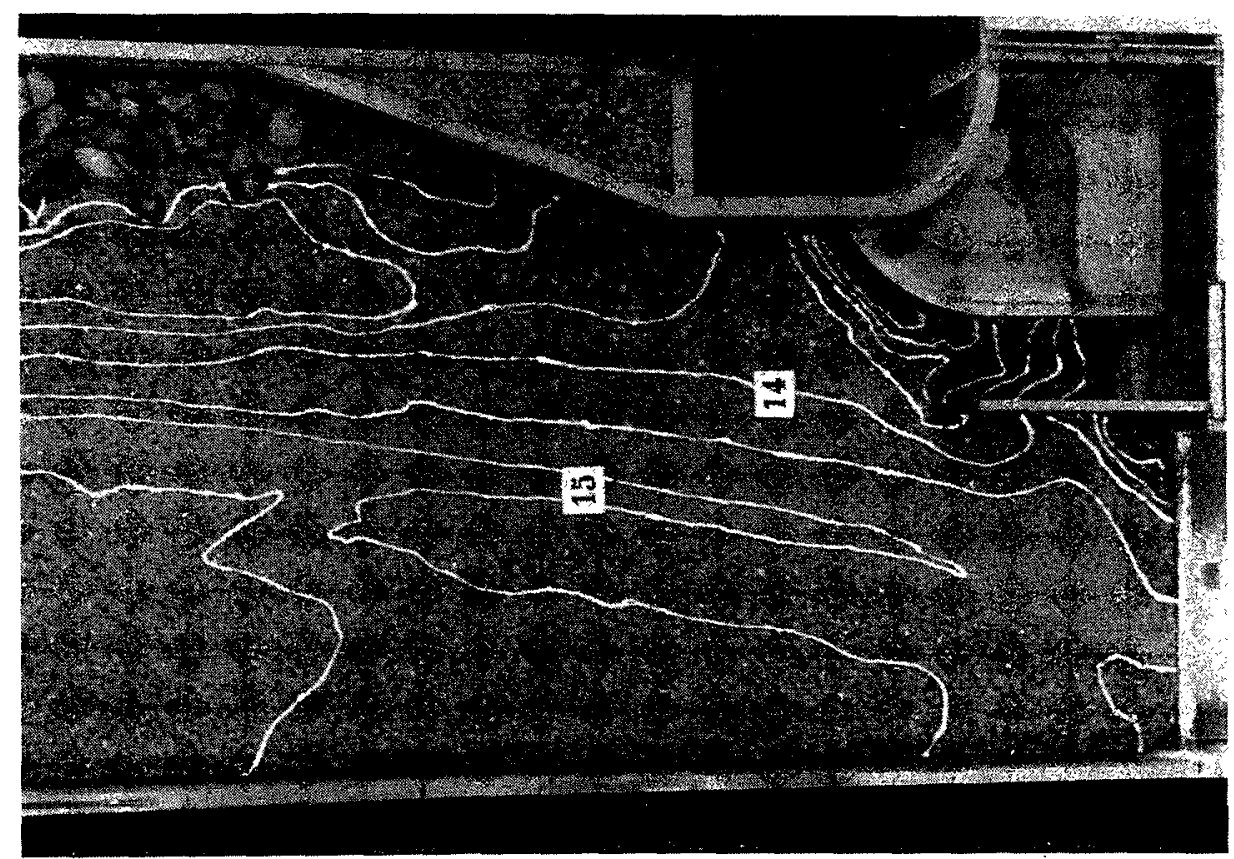

Рното 8. - Expérience 7. Lignes de niveau en fonctionnement normal Répartition du débit liquide :

barrage : $42,9 \%$ - chasse : $31,7 \%$ - prise d'eau : $25,4 \%$

Matériau solide de charriage $\mathrm{n}^{0} 6$

Concentration dans la rivière en amont de la prise d'eau : $0,471 \mathrm{~K}$ 


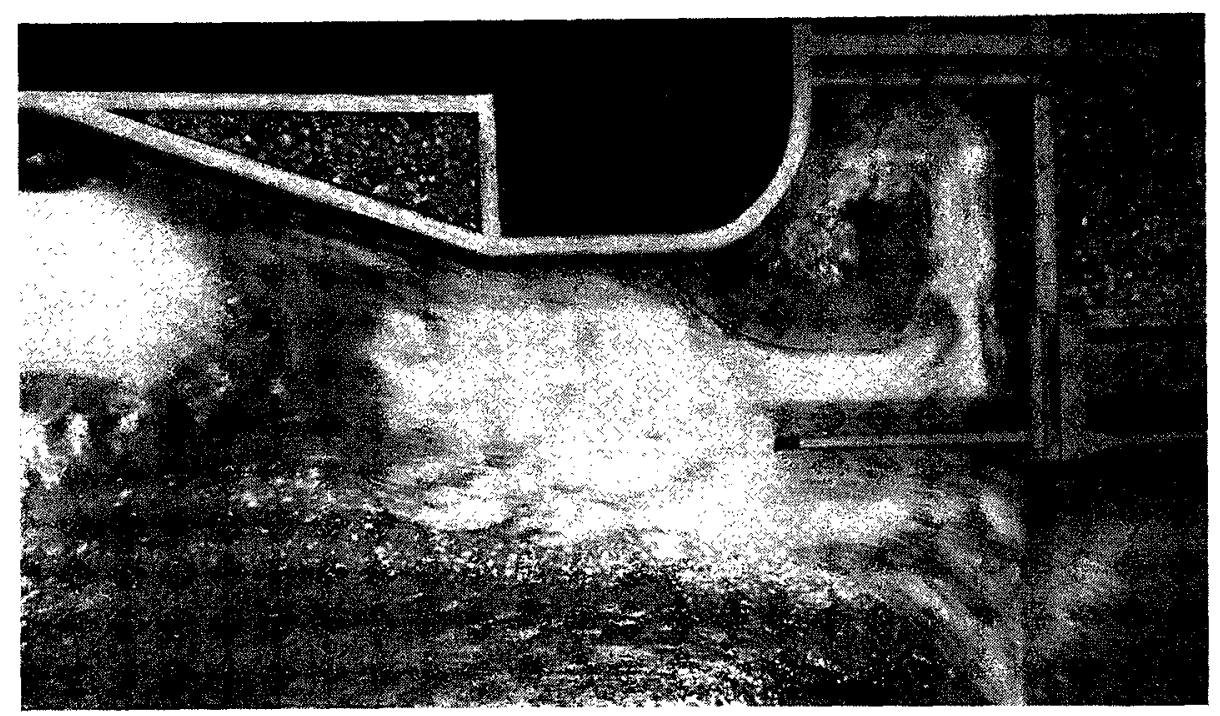

Pното 9. -- Expérience 33. Allure de l'écoulemont en étiage Répartition du débit liquide :

barage : $16,2 \%$-. chasse : $0 \%$ - prise d'ean : $83.8 \%$

relative des débils liméaires dans le canal, le chenal se rapproche de l'axe du canal (photo 7) avec lequel il finit par se confondre (photos 4 et 8). A ce dernier stade, le triage des matériaux du fond dans la rivière s'accentue, disposant des bancs de matériaux fins dont on distingue la couleur plus claire en examinant les résultats d'expériences donnés par les photos 4 et 8 . Le déplacement du chenal principal vers l'axe du canal d'amenée, accompagnant la réduction des débits linéaires au barrage, prépare le chenal d'étiage qui est ainsi à même d'acheminer directement à la prise la plus grande partie du débit de la rivière (photo 9 ).

Si le niveau du plan d'eau au droit du barrage demeure constant, alor's que le débit du canal d'amenée varie, une réduction de ce débit provoque une surélévation des dépôts à l'entrée de ce canal.

4. - Les débits devant pénétrer dans la chambre de prise, ou dans les pertuis de prise s'il n'y a pas de chambre, parcourent obligatoirement le canal d'amenée. En effet, en admettant qu'il y a une chambre de prise, et que le barrage est fixe, ce qui entraine pour la prise les conditions de fonctionnement les plus variables, le seuil de prise peut se trouver submergé ou non, selon le niveau du plan d'eau. Même s'il est submergé, le débit n'a pas d'accès direct à la chambre de prise, ou se produit un grand rouleau périphérique engendré tangentiellemen! par le courant issu de l'échancrure pratiquée à l'extrémité du seuil. Autrement dit, si l'on considère la direction de l'écoulement à la surface du canal d'amenée, en hautes eaux, la chambre de prise constitue une zone délimitant le courant.

Pour perturber cet écoulement, et encore sans rien changer à son allure générale, il faudrait augmenter le débit de la prise jusqu'à dépasser sa capacité normale; même dans ces conditions, on ne décèle qu'une légère inclinaison des filets vers l'intérieur de la chambre, et cela uniquement en aval du tronçon courbe du seuil de prise.

La décantation des matériaux en suspension dans la zone d'eau morte au centre de la chambre modifie légèrement l'allure générale de cet écoulement.

\section{Fonctionnement normal de la prise d'eau}

1. - Par fonctionnement normal, nous entendons le cas où, au cours des crues, les vannes de chasse - ou l'une des vannes si la crue est: légère - restent levées d'une hauteur supérieure à la dimension des plus gros graviers correspondant à la granulométrie des matériaux mis en mouvement. Les vannes de prise d'eau doivent être suffisamment levées pour assurel le débit nécessaire (photo 10).

Le débit empruntant le canal d'amenée se partage entre celui des vannes de chasse, celui de la prise, et une petite fraction qui, franchissant l'extrémité aval de la barbacane, gagne le barrage; cela est dû à une surélévation relative du plan d'eau dans le canal d'amenée par rapport à la surface de la nappe déversant pardessus le barrage, supposé du type fixe.

Le débit pénétrant dans le canal d'amenée est arrêté par l'ouvrage de chasse, ce qui produit 
une surélévation du plan d'eau correspondant a une certaine récupération de l'énergie cinélique. Le pertuis de chasse est alimenté par des filets dirigés vers le bas. La prise reçoit la partie superficielle de l'écoulement qui, après avoir contourné le seuil, est brusquement dévié par l'échancrure vers la dérivation. Le long de la paroi aval de la chambre de prise le plan d'eau est surélevé. Comme on l'a déjà vu, ce mode d'alimentation s'accompagne d'un mouvement de rouleau langentiel qui s'atténue d'ailleurs avec la profondeur; on assure ainsi une répartilion quasi uniforme du débit aux vannes de prise.

2. - Si la rivière charrie des corps flottants, il taul les empêcher de pénétrer dans la prise. Les corps flottants entrés dans le canal d'amenée sont achemines directement vers l'ouvrage de chasse, et s'évacuent vers l'aval en franchissant la partie supérieure déversante des vannes douhles qui doivent être installées en pareil cas.

Si un corps flottant pénètre dans la chambre de prise, il se trouve entraîné par le courant langentiel de retour. En très hantes eaux, le seuil de prise peut être submergé; la turbulence superficielle du courant de retour est capable de ramener le corps flottant vers le courant principal du canal d'amenée, et de lui donner ainsi une nouvelle occasion de franchir les ouvrages vers l'aval.

3. - La configuration des dépôts dans le canal d'amenée dépend des fluctuations du plan d'ean et de la répartition du débit entre le barrage, les ouvrages de chasse et la prise.

Pour des prises d'eau de mêmes caractéristiques et placées dans les mêmes conditions de fonctionnement, mais en faisant varier la granulométrie spécifique de la rivière, on constate des changements dans la configuration des dépôts formés à l'entrée et à l'intérieur du canal d'amenée (photos $3,4,8$ ). Au fur et à mesure que le débit linéaire du canal d'amenée augmente par rapport à celui du barrage, le volume des matériaux charriés pénétrant dans le canal augmente également.

Dans chaque cas de fonctionnement, la surface des dépôts atteint un profil d'équilibre lié au courant qui entraine les matériaux charriés dans le canal d'amenée et de là, à travers les ouvrages de chasse, dans la rivière à l'aval.

Généralement, la surface de ce dépôt s'abaisse progressivement dans le canal d'amenée jusqu'au voisinage du pertuis de chasse où il se termine en talus. Le rouleau se produisant au pied de ce talus le maintient en place avec une configuration qui dépend du niveau du plan d'eau et du mode de répartition des débits. Ces conditions demeurant constantes, les matériaux qui continuent à pénétrer dans le canal d'amenée sont charriés dans la couche liquide surmontant immédiatement la surface du dépôt. et sont finalement chassés vers l'aval.

Le courant qui franchit les ouvrages de chasse présente des zones à charriage plus on moins

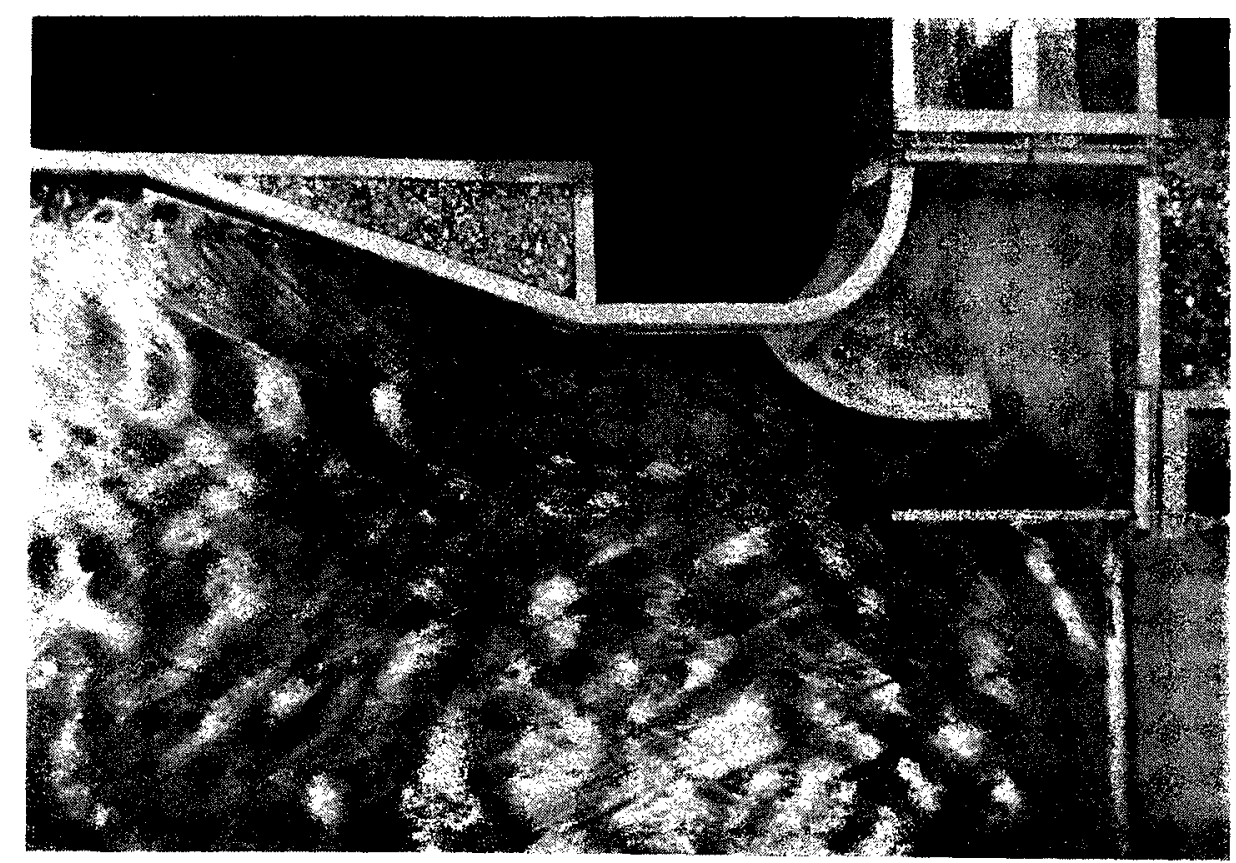

Phoro 10. - Lxpérience 30. Allure de l'écoulement en fonctionnement normal Répartition du débit liquide : barrage : $65,2 \%$ - chasse : $19,3 \%$ - prise d'eau : $15,5 \%$ 
intense; ces zones varient selon les cas de fonctionnement. C'est seulement lorsque le débit de charriage évacué est très élevé que sa distribution est bien uniforme le long des vannes.

4. - On a vu au paragraphe 2 du chapitre précédent les phénomènes d'érosion locale provoqués par le tronçon courbe du seuil de prise et par l'extrémité amont du mur guideau; on a également analysé les mouvements des matériaux charriés entrainés dans ces zones d'érosion.

Très souvent, l'érosion à l'extrémité du mur guideau ne se produit pas, ou est très faible et demeure indépendante de celle qui règne le long du seuil de prise. Lorsque les matériaux sont fins et les débits linéaires relativement élevés dans le canal d'amenée, l'érosion due au seuil de prise se prolonge dans le canal d'amenée jusqu'à atteindre parfois l'échancrure. Dans ces conditions, les dépôts dans le canal d'amenèe ont une configuration dissymétrique et sont plus épais le long du mur guideau (photos 4 et 7).

Les matériaux entraînés dans ce fossé d'érosion sont triés, les plus gros tombant au fond. Le profil d'équilibre une fois atteint, les matériaux se déplacent le long $d u$ fossé, principalement au pied du talus; finalement ils parcourent les dépôts du canal d'amenée, puis sont chassés vers l'aval.

5. - Sur une petite longueur, dans sa partie amont, la barbacane laisse passer un faible débit vers le canal d'amenée; sur le restant de sa section, l'écoulement est par contre dirigé vers le barrage.

TABLEAU I

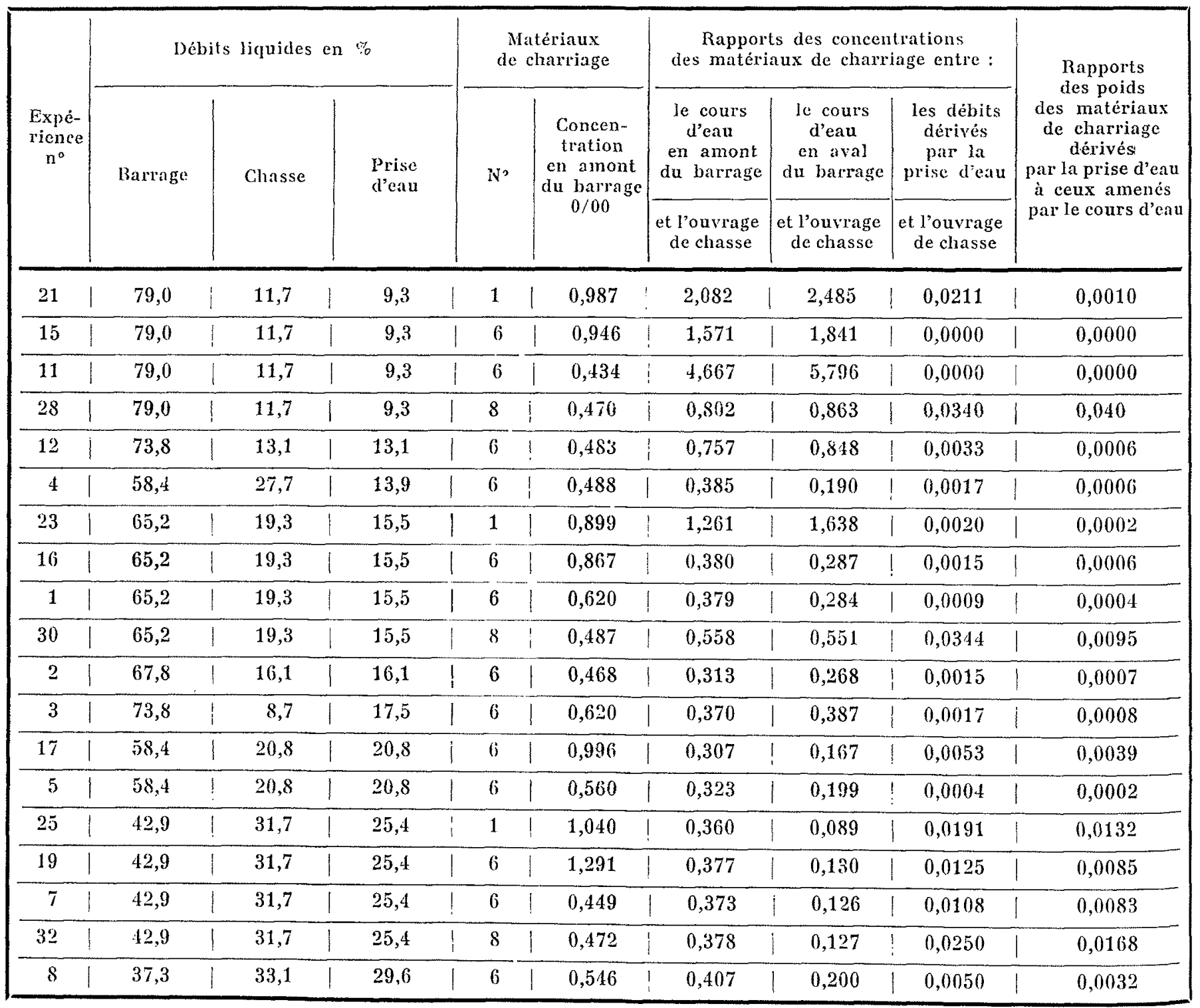


Dans le cas particulier où la courbe granulométrique révèle une prédominance des éléments fins, et où le débit linéaire du barrage est petit, quelques éléments parviennent sur le talus des dépôts du canal d'amenée et sortent par l'ouvrage de chasse.

Généralement, avec des granulométries plus grosses, quelques éléments fins pénètrent dans la portion amont de la barbacane, mais avant de tomber dans le canal d'amenée, le courant prédominant les renvoie à la rivière.

En fonctionnement normal, les dépôts du canal d'amenée demeurent presque toujours en dessous du seuil de la barbacane; il est presque impossible, dans ces conditions, qu'un mouvement de charriage vers la rivière se produise à travers celte ouverture.

6. - Le seuil de l'échancrure reste au-dessus les dépôts du canal d'amenée, quelles que soient les fluctuations du plan d'eau et la répartition du débit entre les ouvrages de chasse et la prise.

Ainsi, l'accès du malériau charrié dans la prise est-il impossible.
7. - Le tableau I donne, par rapport à la concentration en matériaux charriés passant dans les ouvrages de chasse, les concentrations correspondant aux apports de l'amont, à la rivière en aval du barrage et au débit dérivé; de plus, ce tableau précise le rapport entre le poids total de matériaux amenés par la rivière et le poids des matériaux dérivés par la prise pendant un intervalle de temps donné.

La figure 1 fait apparaitre les valeurs relatives des rapports entre concentrations des débits dérivés et concentrations des débits de chasse; pour chaque point de ce graphique sont indiqués : la répartition du débit entre le barrage, les chasses et la prise, le numéro de repère du matériau de charriage utilisé, et la concentration dans la rivière en amont.

La figure 2 met en évidence les valeurs relatives des rapports entre poids des matériaux dérivés et poids des matériaux amenés par la rivière dans un même laps de temps; pour chaque point, sont en outre donnés les mêmes renseignements que dans la figure 1. Ces deux

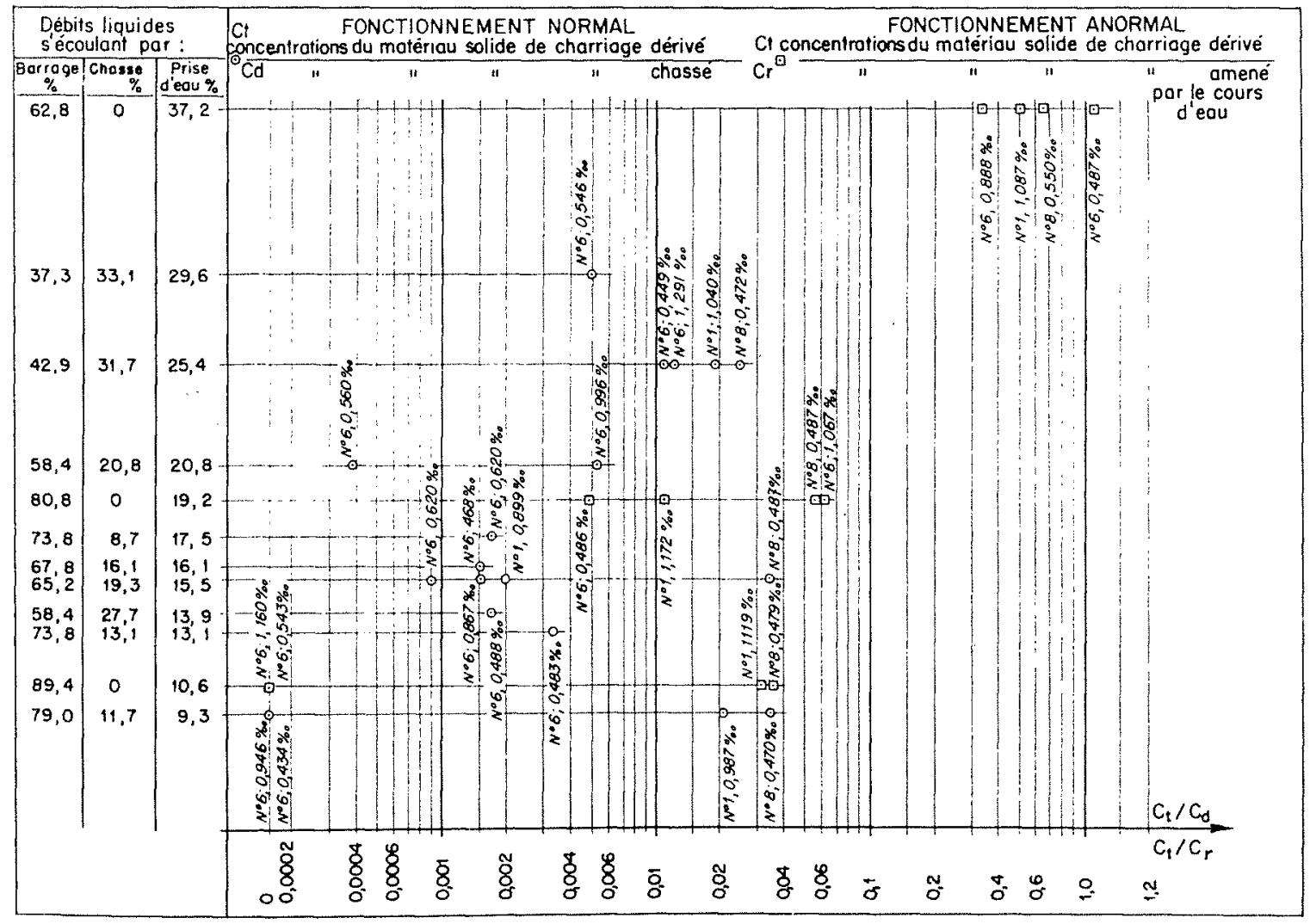

Fic. 1. - Fonctiomnement normal, Rapports entre les concentrations du materiau solide de charriage dérivé par la prise d'eau et celui chassé. Fonctionnement anormal. Rapports cntre les concentrations du matériau solide de charriage délivré par la prise d'eau et celui amené par le cour's d'eau. 


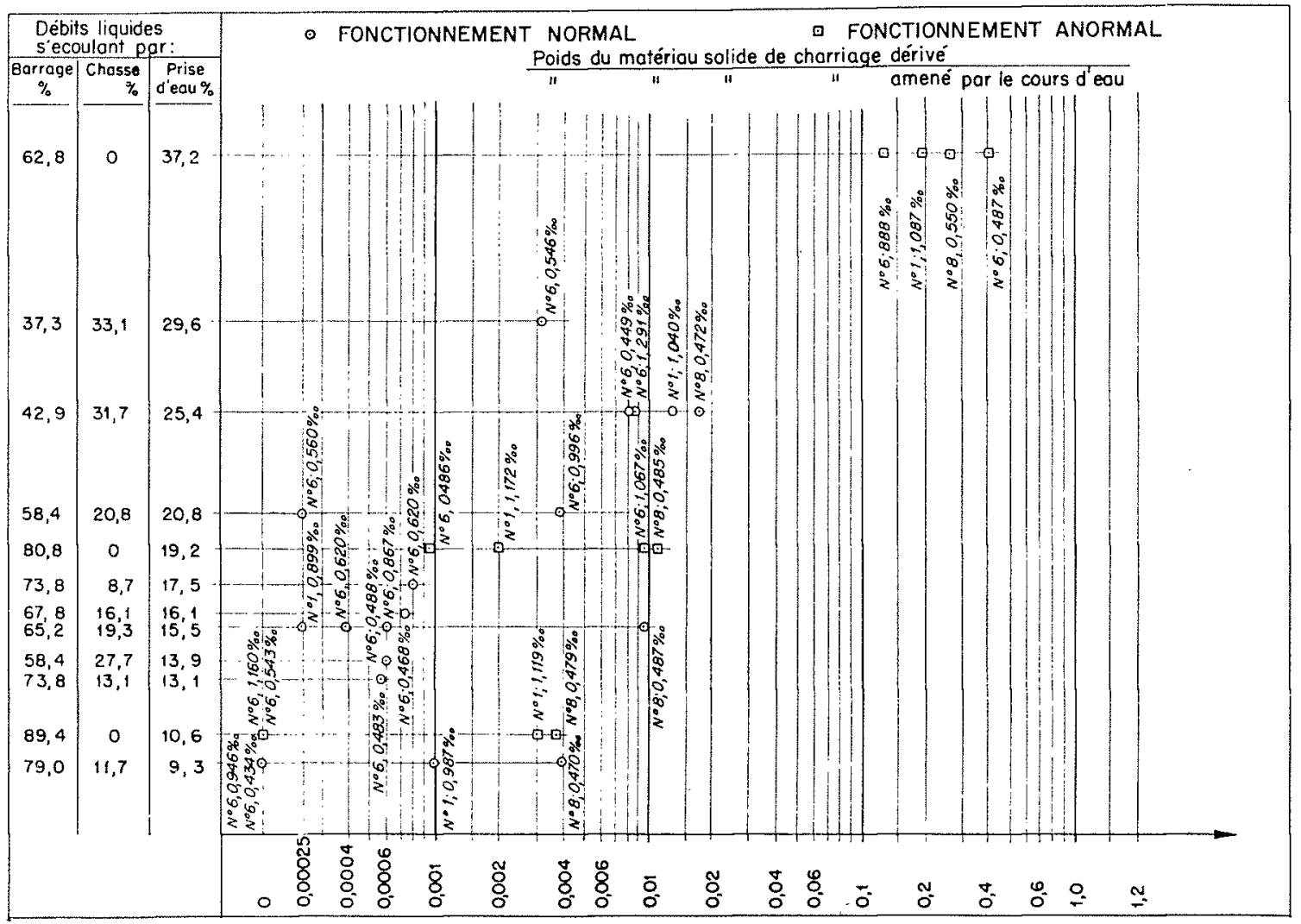

Fis. 2. - Rapports entre le poids du matériau solide de charriage dérivé par la prise d'eau et celui amené par le cours d'eau.

figures révèlent des ressemblances assez frappantes.

L'examen du tableau I et des figures 1 et 2 montre que les valeurs relatives caractérisant la concentration et le volume des matériaux dérivés, augmentent au fur el à mesure qu'augmente le débit linéaire du canal d'amenée par rapport à celui du barrage, c'est-à-dire au cours d'une période de décrue. Rappelons cependant que la capacité de charriage d'un cours d'eau diminue fortement lorsque le débit liquide diminue, et qu'elle tend à s'annuler; il en résulte, dans ces conditions, que la quantité absolue des matériaux dérivés est insignifiante.

Dans le cas le plus défavorable réalisé au cours de nos essais, la prise dérive $16,8 \%$ du volume des apports, particulièrement fins, de la rivière; le rapport correspondant des concentrations est 0,066 .

On peut indiquer, à titre de comparaison, que dans les prises d'eau classiques, ce rapport a des valeurs variant de 0,5 à plus de 1 , à l'exception de celles équipées d'un seuil muni à la base d'orifices de dégravement à entrée normale; ceux-ci limitent la hauteur des dépôts en évacuant à l'aval les apports solides de charriage de la rivière.
Les matériaux charriés pénètrent dans la prise après avoir été mis en suspension par les tourbillons qui se forment dans le canal d'amenée au niveau de l'extrémité amont de l'échancrure. Dans le cas de l'essai 21 (photo 5), la chambre de prise s'est trouvée alimentée par l'extrémité amont de la partie courbe $d u$ seuil de prise; il s'agissait d'une crue importante caractérisée par de fortes concentrations et une grosse granulométrie : les dépôts s'accumulent alors sur une grande épaisseur à l'entrée du canal d'amenée et, par suite de la saltation, des matériaux franchissent le seuil de prise.

Dans chaque cas particulier d'application, il est toujours possible, au moyen de dispositions simples, de réduire à des valeurs insignifiantes ou nulles la concentration en matériaux charriés du débit dérivé. Soulignons que les essais du modèle final ont toujours porté sur les mêmes configurations, qui ont été soumises à des conditions extrêmement variables sans qu'y soient introduits aucune modification ou aucun perfectionnement susceptible de conduire à de meilleurs résultats.

8. - Les différentes expériences révèlent qu'il se produit un triage des matériaux charriés entre 
le barrage, les ouvrages de chasse et la prise d'eau (fig. 3).

Pour les débits abondants et des rivières à

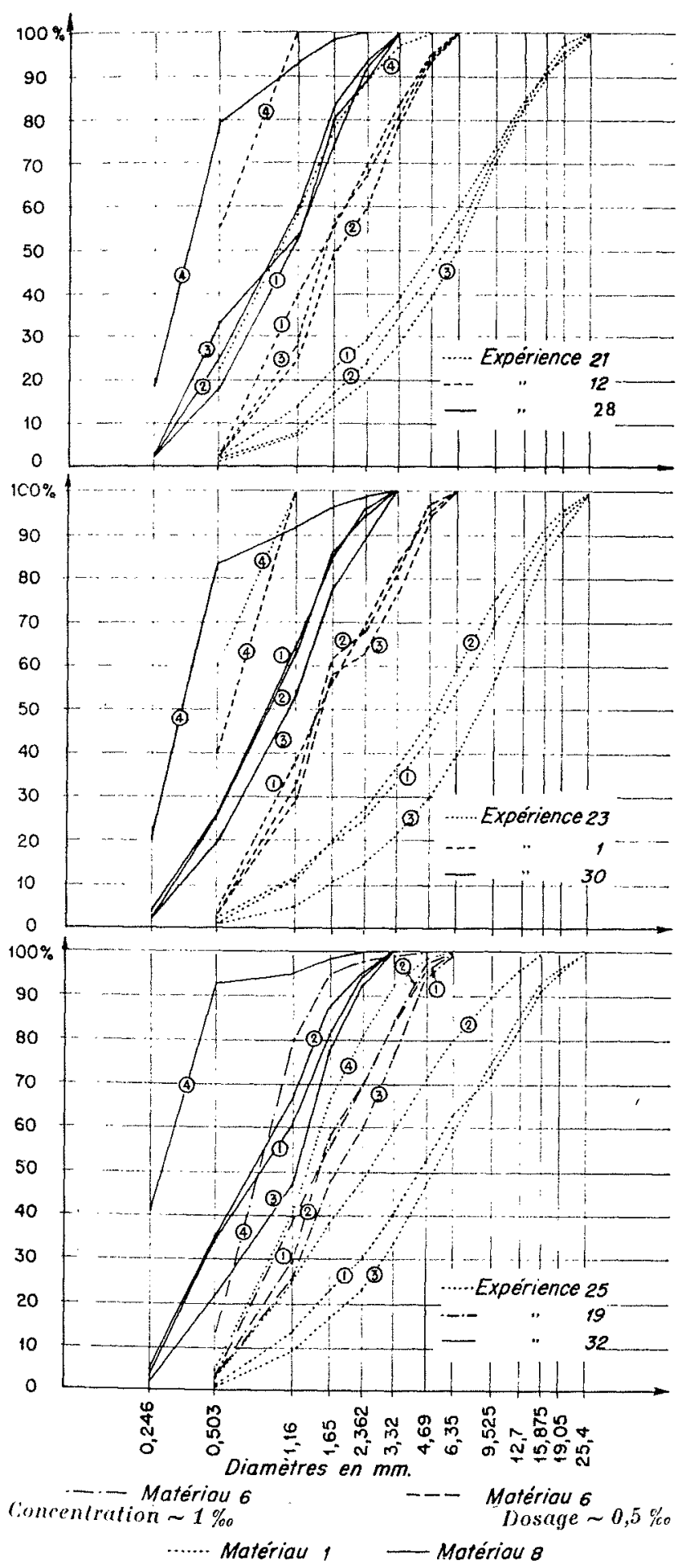

Fic. 3. - Fonctionnement normal.

Courbes granulométriques des matériaux solides de charriage.

(1) Rivière en amont du barrage;

(2) Rivière en aval du barrage;

(3) Ouvrage de chasse

(4) Prise d'eau. grosses granulométrie, les matériaux évacués en aval par le barrage et les chasses présentent une granulométrie plus élevée que ceux amenés à l'amont; lorsque la granulométrie des apports est plus petite, les trois courbes granulométriques sont assez voisines.

Dans le cas des débits de crue moyens et. faibles, la granulométrie des matériaux évacués par les chasses est plus grosse que celle des apports de la rivière. Les courbes granulométriques caractérisant la rivière à l'amont et à l'aval sont très semblables; c'est seulement dans le cas où l'on se trouve en présence à la fois de petits débits et d'une grosse granulométrie des apports de la rivière, que la granulométrie à l'aval du barrage est plus fine qu'à l'amont.

Les courbes granulométriques relatives aux quelques matériaux de charriage pénétrant dans la prise montrent que ceux-ci sont constituís des grains les plus fins; on voit sur la figure 3 que les courbes granulométriques $n^{\circ} 4$, correspondant à la prise, sont constamment placées à gauche de celles correspondant aux apports de la rivière.

Par leurs dimensions, ces grains s'apparentent aux matériaux transportés en suspension dans la rivière; ceux qui pénètrent dans la dérivation poursuivront aisément leur chemin jusqu'au dessableur où ils seront éliminés.

\section{Fonctionnement en étiage}

1. - Sous l'influence de l'affaiblissement du débit, le chenal principal dirigé vers le canal d'amenée tend à s'accuser.

Lorsque le débit liquide s'abaisse, le débit de charriage s'abaisse aussi; et - le matériau d'essai demeurant inchangé - les courbes granulométriques relevées montrent que ce chirriage porte sur des grains de plus en plus fins. Ainsi, ses rannes à peine ouvertes, l'ouvrage de chasse est-il à même d'évacuer les très faibles excédents de débit, sans risquer de retenir des cailloux pouvant former bouchon.

De cette facon, le fonctionnement normal des ouvrages maintient le chenal principal dirisé vers le canal d'amenée dans lequel l'épaisseur des dépòts reste limitée.

Finalement, les débits d'étiage sont presque totalement dirigés vers la prise (photo 9). Dans le cas du harrage fixe, la faible portion de ces débits se dispersant dans d'autres directions se rassemble en un courant qui longe le harrage et pénètre dans le canal d'amenće par la harbacane du mur guideau. Dans le cas du barrage mobile, il est usuel et aisé de rassembler les eaux dispersées et de les diriger rers le canal d'amence. 


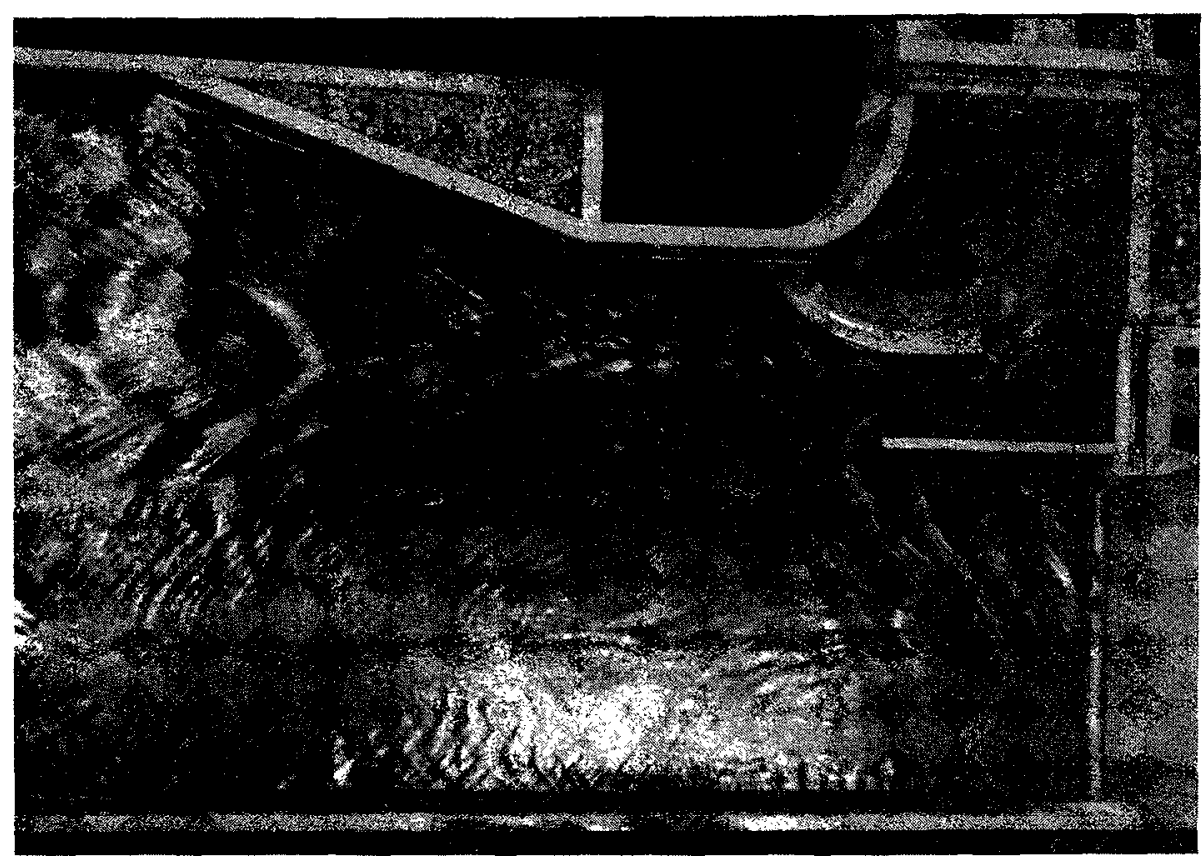

Photo 11. - Expérience 34. Allule de l'écoulement en fonctionmement anormal Répartition du débit liquide :

barrage : $62,8 \%$ - chasse : $0 \%$ - prise d'eau : $37,2 \%$

2. - Pendant les plus basses eaux, les vannes de chasse doivent rester fermées. Les vannes de prise doivent être suffisamment levées pour que soit dérivée la totalité du débit disponible ou tout au moins le débit nécessaire.

En étiage, certaines rivières charrient du sable et du limon, tout en restant limpide; ceci provient du triage qui s'opère sur le fond par suite des déplacements du matériau fin, et qui a tendance à provoquer la formation de rigoles tapissées de graviers et de cailloux.

Dans de telles rivières, ces apports sablolimoneux surélèvent les dépôts dans le canal d'amenée jusqu'au niveau du seuil de l'échancrure; une fois ce niveau atteint, les nouveaux apports pénètrent dans la dérivation jusqu'au dessableur.

Afin d'éviter la consolidation des dépôts contre les vannes de chasse, il est recommandé de manœuvrer celles-ci périodiquement pendant la courte durée nécessaire à l'entraînement du banc de sable.

\section{Fonctionnement anormal de la prise d'eau}

1. - Dans les rivières torrentielles, les crues peuvent se déplacer vers l'aval sous forme d'ondes faisant passer brutalement du régime d'étiage à un rẻgime de très hautes eaux. Dans ces conditions, elles peuvent surprendre les vannes de chasse fermées; c'est ce que nous entendons par fonctionnement anormal (photo 11).
En présence d'un accroissement inattendu du débit, ce type de prise d'eau fonctionne suffsamment bien pour continuer à assurer néanmoins, sur une période assez longue, une dérivation des eaux n'entraînant qu'un volume très réduit de matériaux de charriage.

Il est impossible de songer à laisser la prise sans surveillance; aucun ouvrage de ce genre ne peut fonctionner correctement en l'absence d'un bon service technique d'exploitation.

Il est évident que, dans le cas d'un barrage mobile, il conviendra de jouer sur toutes ses vannes.

Dans le cas d'un barrage fixe, l'élévation du plan d'eau se traduit par une augmentation du débit dérivé vers le canal; en outre, les dépôts se trouvent progressivement surélevés jusqu'à l'établissement d'un nouveau profil d'équilibre sur lequel s'amorce à nouveau le phénomène de charriage. Au cours de cette évolution, la quantité de matériaux charriés parvenant à la prise ou franchissant le barrage est insignifiante.

Nos observations ont été effectuées une fois terminée la période transitoire, dont la durée dépend des caractéristiques de l'onde de crue, et par conséquent une fois terminé le processus d'évolution du fond; autrement dit, les observations concernant le fonctionnement anormal ont été faites en présence de conditions d'écoulement permanentes. Ceci correspond à une éventualité qui ne doit pas se produire; cependant, il est bon de signaler que, même dans ces conditions anormales, la prise étudiée fonctionne assez bien. 


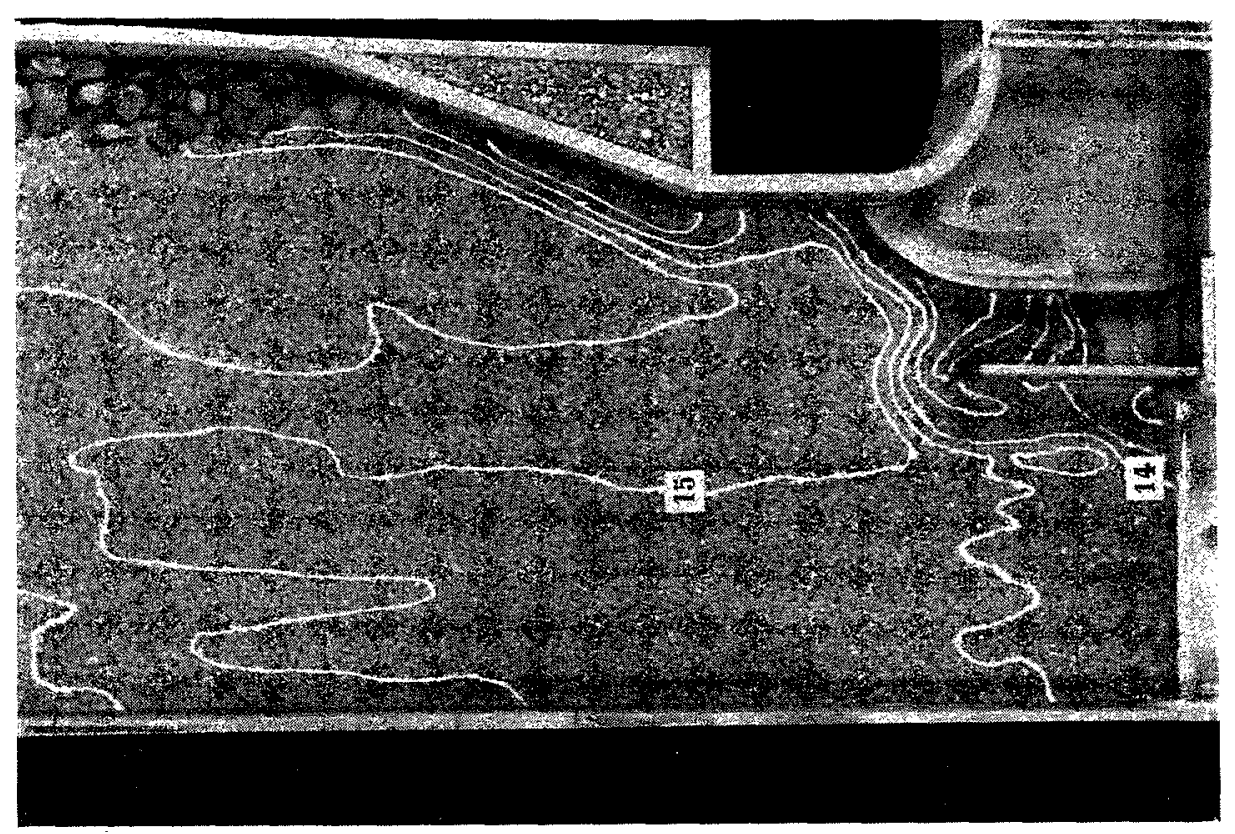

Рното 12. - Expérience 13. Lignes de niveau en fonctionnement anormal Répartition du débit liquide :

barrage : $89,4 \%$ - chasse : $0 \%$ - prise d'eau : $10,6 \%$

Matériau solide de charriage $n^{\circ} 6$

Concentration dans la rivière en amont de la prise deau : $0,543 \%$

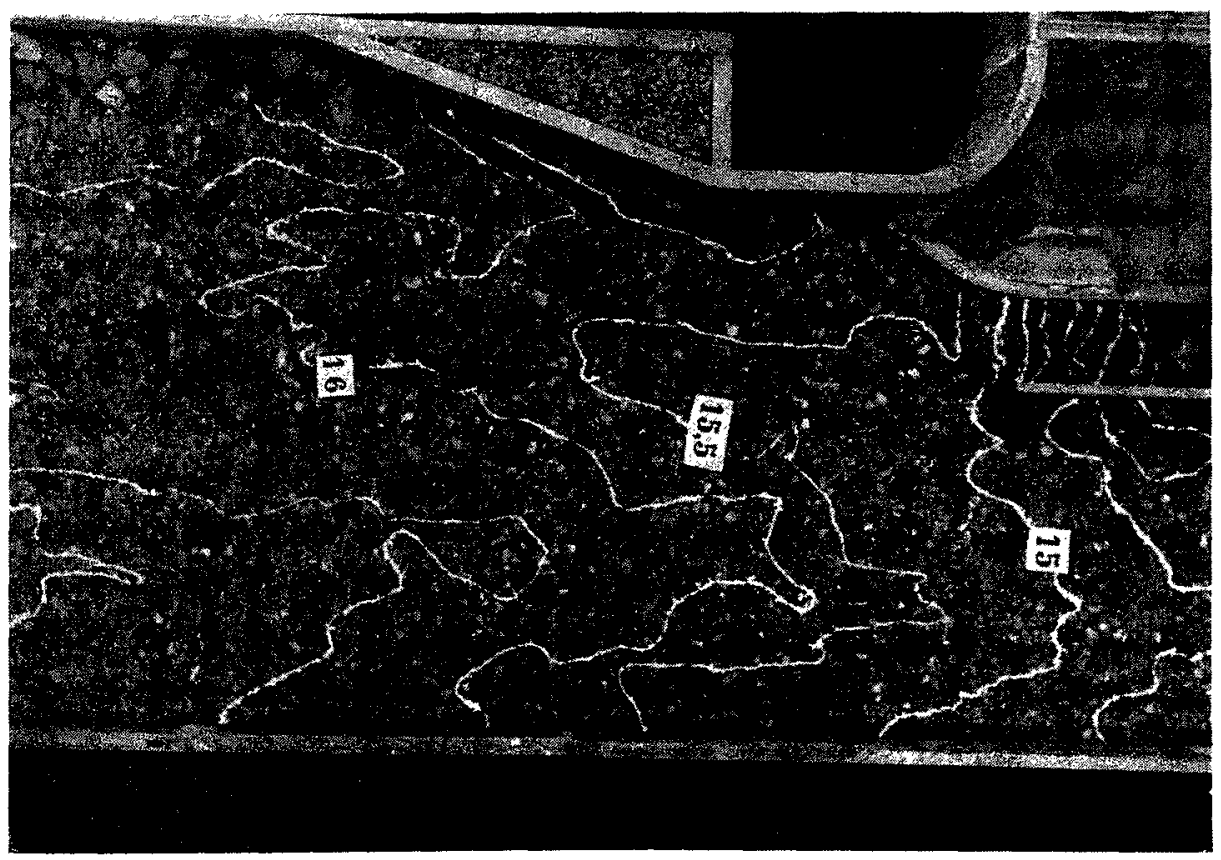

Pнoto 13. - Expérience 22. Lignes de niveau en fonctionnement anormal Répartition du débil liquide :

l)arrage : $89,4 \%$ - chasse : $0 \%$ - prise d'eau : $10,6 \%$

Matériau solide de charriage. $n^{0} 1$

Concentration dans la rivière en amont de la prise d'eau : $1,119 \%$ 


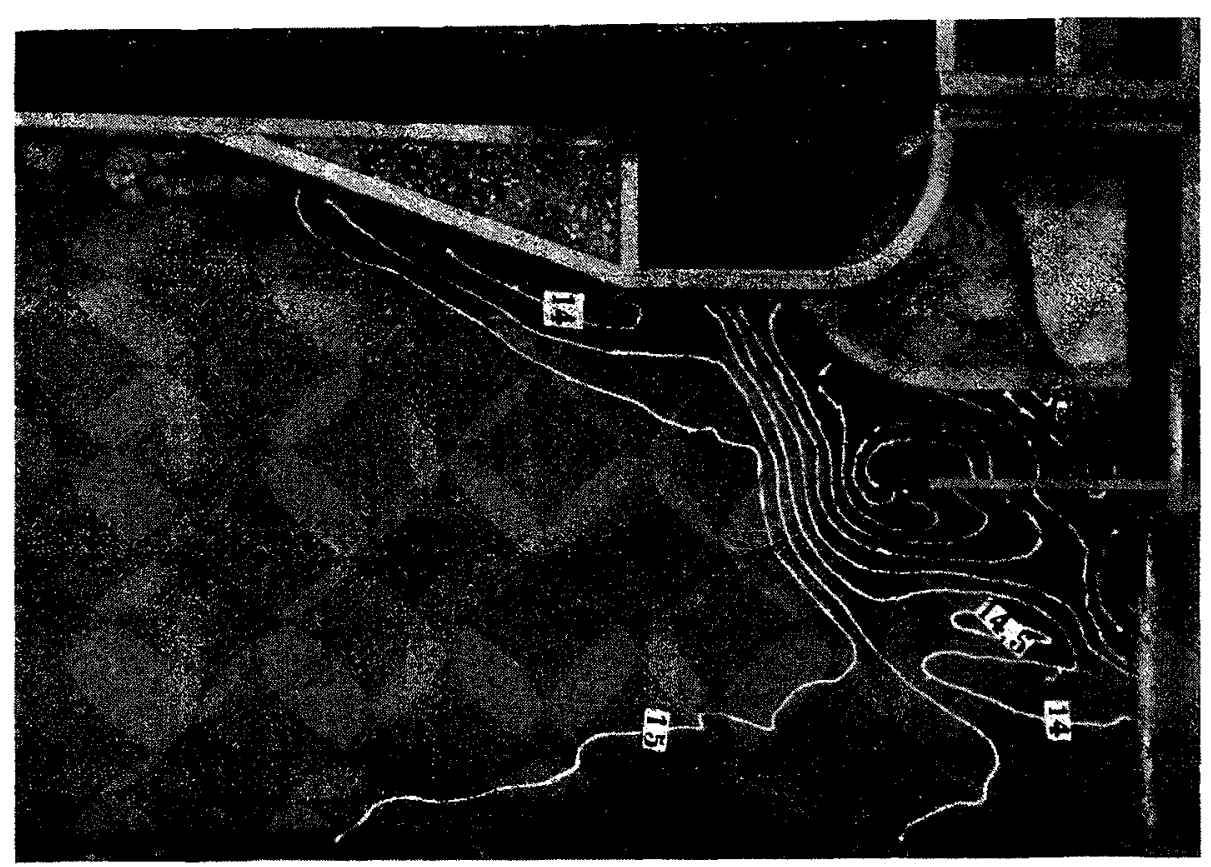

Photo 14. - Expérience 29. Lignes de niveau en fonctionnement anormal Répartition du débit liquide :

barrage : $89,4 \%$ - chasse : $0 \%-$ - prise d'enu : $10,6 \%$

Matériau solide de charriage $n^{\circ} 8$

Coucentration dans la rivière en amont de la prise d'eau : $0,479 \%$

\section{TABLEAU II}

\begin{tabular}{|c|c|c|c|c|c|c|c|}
\hline \multirow{3}{*}{$\begin{array}{l}\text { Expérience } \\
n^{\circ}\end{array}$} & \multirow{3}{*}{$\begin{array}{c}\text { Rapports } \\
\text { des débits liquides } \\
\text { linćaires entre } \\
\text { barrage et } \\
\text { (ainal d'alimentation }\end{array}$} & \multicolumn{3}{|c|}{$\begin{array}{l}\text { Matèriaux } \\
\text { de charriage }\end{array}$} & \multicolumn{2}{|c|}{$\begin{array}{l}\text { Rapports des concentrations } \\
\text { des matériaux de charriage entre: }\end{array}$} & \multirow{3}{*}{$\begin{array}{l}\text { Rapports des poids } \\
\text { des matériaux } \\
\text { de charriage } \\
\text { dérivés par } \\
\text { la prise d'eau } \\
\begin{array}{c}\text { à ceux amenés } \\
\text { par le cours d'eau }\end{array}\end{array}$} \\
\hline & & \multirow{2}{*}{$\mathrm{N}^{0}$} & \multicolumn{2}{|c|}{$\begin{array}{l}\text { Concen- } \\
\text { lration } \\
\text { en amont } \\
\text { du barage } \\
0 / 00\end{array}$} & $\begin{array}{c}\text { le cours d'eau } \\
\text { en aval du barrage }\end{array}$ & \multirow{2}{*}{$\mid \begin{array}{c}\text { ceux dérivés } \\
\text { par la prise d'eau }\end{array}$} & \\
\hline & & & & & $\begin{array}{l}\text { et le cours d'eau } \\
\text { en amont du barrage }\end{array}$ & & \\
\hline 22 & 2,50 & 1 & & 1,119 & 1,114 & 0,0310 & 0,0030 \\
\hline 14 & 2,50 & 6 & & 1,160 & 1,118 & 0,0000 & 0,0000 \\
\hline 13 & 2,50 & 6 & & 0,543 & 1,118 & 0,0000 & 0,0000 \\
\hline 29 & 2,50 & 8 & 1 & 0,479 & 1,114 & 0,0346 & 0,0037 \\
\hline 24 & 1,25 & 1 & i & 1,172 & 1,235 & 0,0110 & 0,0020 \\
\hline 18 & 1,25 & 6 & i & 1.067 & 1,223 & 0,0610 & 0,0097 \\
\hline 6 & 1,25 & 6 & i & 0,486 & 1,235 & 0,0049 & 0,0009 \\
\hline 31 & 1,25 & 8 & 1 & 0,485 & 1,224 & 0,0557 & 0,0107 \\
\hline 27 & 0,50 & 1 & 1 & 1,087 & 0,210 & 0,5190 & 0,1930 \\
\hline 20 & 0,50 & 6 & 1 & 0,888 & 1,393 & 0,3370 & 0,1280 \\
\hline 10 & 0,50 & 6 & $!$ & 0,487 & 0,906 & 1,1600 & 0,3940 \\
\hline 34 & 0,50 & 8 & i & 0,550 & 1,197 & 0,6669 & 0,2479 \\
\hline
\end{tabular}


2. - Les eaux qui s’écoulent dans le canal d'amenée se heurtent aux vannes de chasse fermées, leur niveau s'élevant en raison de la l'éduction de l'énergie cinétique; au voisinage du fond, un courant de retour se produit, en parliculier au pied du talus des dépôts.

La prise reçoit les eaux qui s'écoulent en surlace el longent le seuil de prise : celles-ci sont brusquement déviées à travers l'échancrure, vers la chambre, contre la paroi de laquelle elles subissent une certaine surélévation. Ce mode d'alimentation s'accompagne d'un mouvement de rouleau langentiel qui s'atténue d'ailleurs avec la profondeur; on assure ainsi une réparlilion suffisamment uniforme du débit aux vannes de prise.

3. - Lorsque les débits linéaires du barrage sont supérieurs à ceux du canal d'amenée, le chenal principal se déplace vers la retenue; à l'imuont du canal d'amenée, les dépôts s'engraissent des plus gros matériaux de charriage qui ne pouvent plus êlre entraînés.

On obtient ainsi que les matériaux charriés se dirigeant vers l'entrée du canal se dévient vers la zone de la retenue bordée par le mur guideau (photos 12 et 13 ).

Les rivières dont le matériau de fond est fin domnent lieu à des affouillements intenses à l'entrée du canal d'amenée; les grains tombant dans la fosse sont entraînés par le mouvement hélicoïdal au-delà du musoir du mur guidean, puis sont évacués à l'aval par le barrage; on empêche ainsi, ou du moins on limite au maximum, l'entrée des matériaux dans le canal d'amenée. Dans ces conditions, la courbure du seuil de prise prolonge ce travail d'affouillement à l'intérieur du canal d'amenée dont les dépôts prennent une disposition dissymétrique (photos 12 et 14 ).

Avec la répartition des débits indiqués plus haut, le reflux des eaux du canal d'amenée empêche que l'intensité des apports solides atteigne une valeur compromettant le maintien de la surface des dépôts à une cote assez faible.

Pour les valeurs 2,50 et 1,25 du débit linćaire du barrage par rapport au débit linéaire du canal d'amenée, et en précisant dans chaque cas la granulometrie et la concentration adoptées, le tableau II donne :

a) le rapport entre les concentrations à l'aval et à l'amont du barrage;

b) le rapport entre la concentration dans la dérivation el celle à l'amont du barrage;

c) le rapport entre poids de matériaux dérivés el poids des matériaux amenés par la rivière.

I.es figures 1 et 2 donnent les valeurs de $b$ ) et

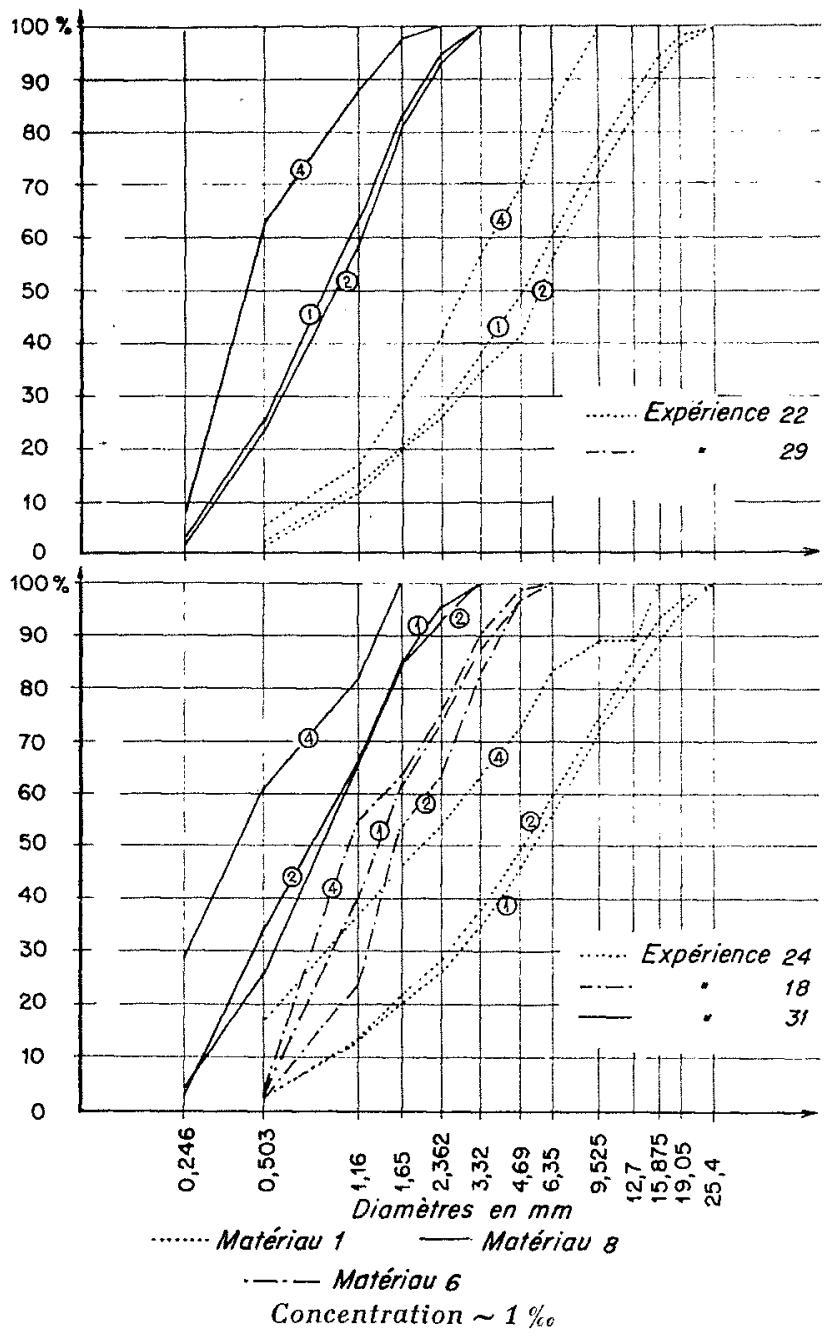

Fic. 4. - Fonctionnement anormal.

Courbes granulometriques des matériaux solides de charriage.

(1) Rivière en amont du barrage;

(2) Rivière en aval du barrage;

(4) Prise d'eau.

c) correspondant respectivement à la répartition suivante des débits :

Barrage : 89,4\%; prise d'eau : $10,6 \%$

Barrage : $80,8 \%$; prise d'eau : $19,2 \%$

La représentation graphique de ces valeurs montre qu'elles restent plus ou moins à l'intérieur des limites des résultats trouvés dans le cas du fonctionnement normal.

La figure 4 fait bien apparaitre que, de l'amont à l'aval du barrage, il ne se produit pratiquement pas de triage dans la riviere. Par contre, les quelques matériaux de charriage pénétrant dans la prise d'eau subissent un triage sévère sélectionnant la portion la plus fine de la granulonétrie. 


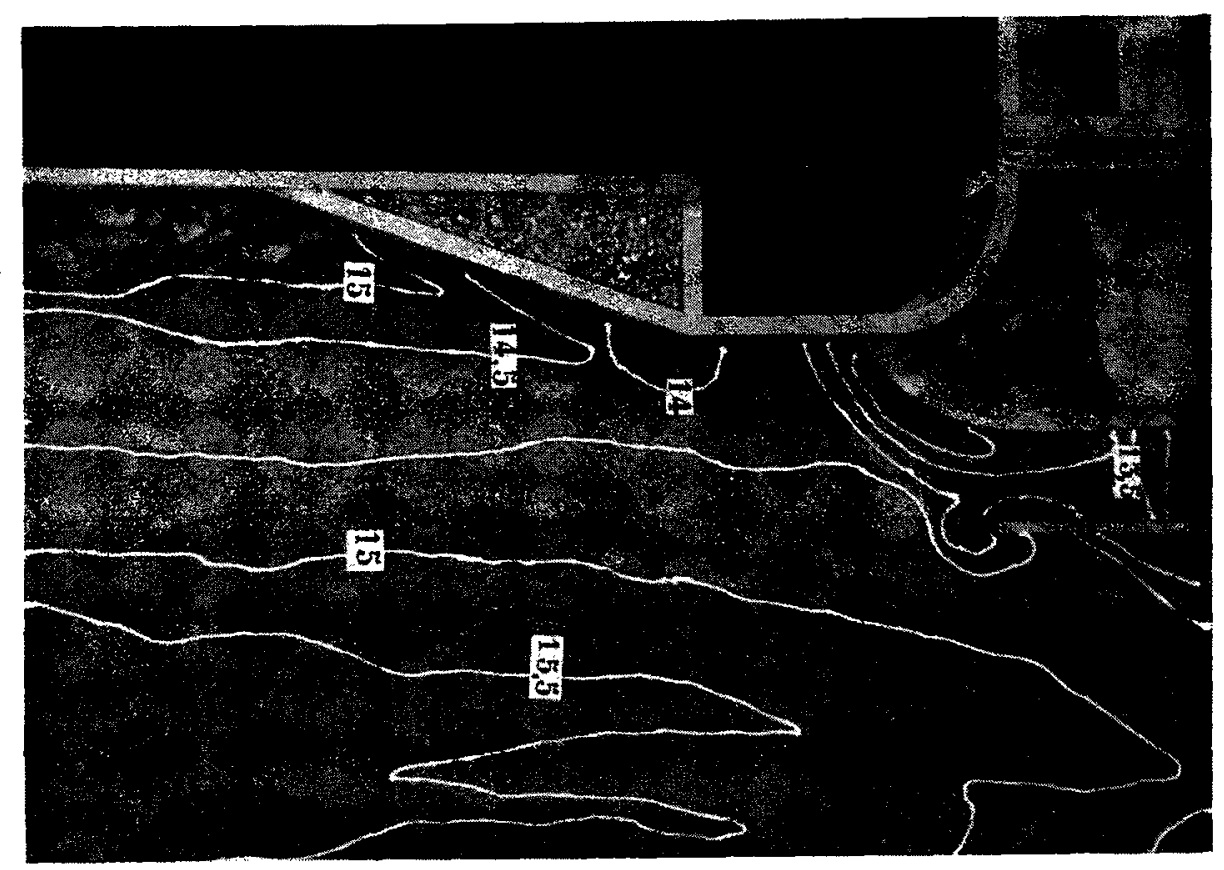

Рното 15. - Expérience 34. Lignes de niveau en fonctionnement anormal Répartition du débit liquide :

barrage : $62,8 \%$ - chasse : $0 \%$ - prise d'eau : $37,2 \%$

Matériau solide de charriage $n^{\circ} 8$

Concentration dans la rivière en amont de la prise d'eau : $0,550 \%$

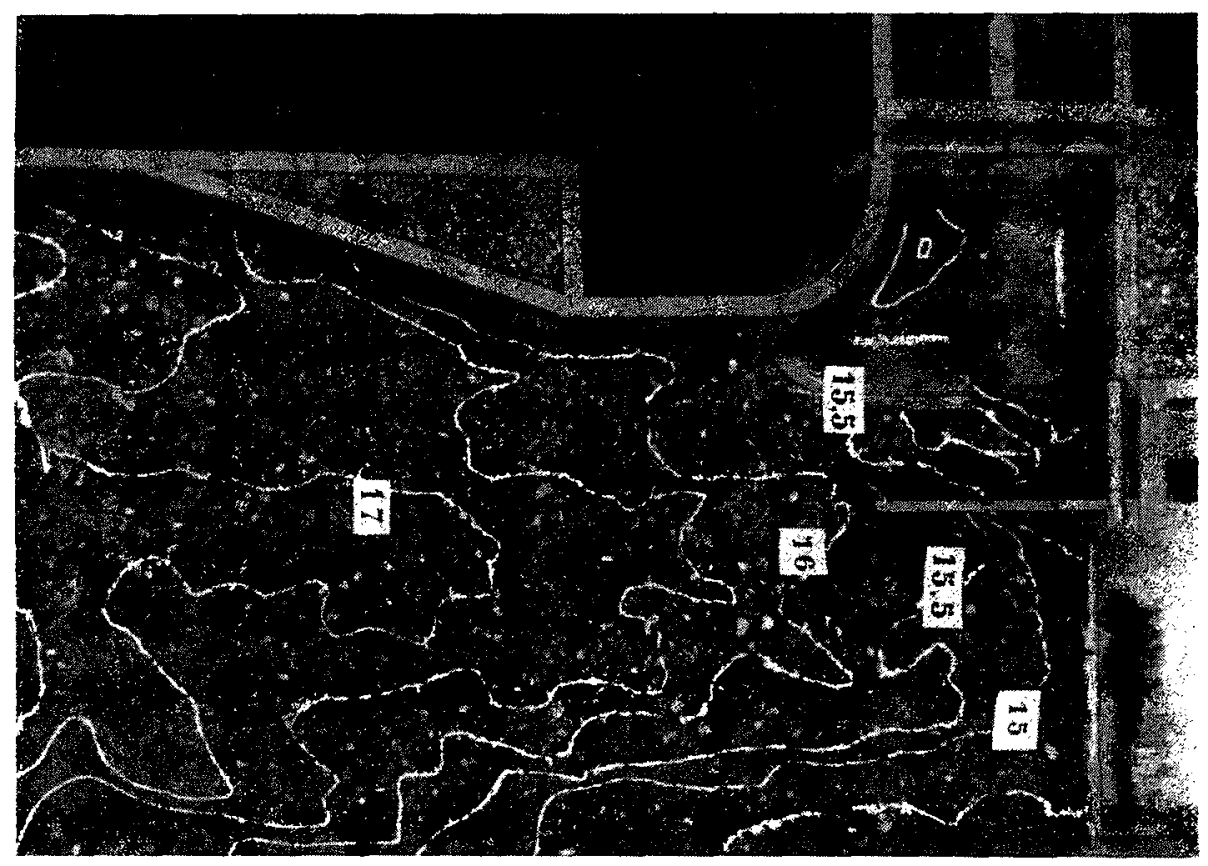

Pнoro 16. - Expérience 27. Lignes de niveau en fonctionnement anormal Répartition du débit liquide :

harrage : $62,8 \%$ - chasse : $0 \%$ - prise d'eau : $37,2 \%$

Matériau solide de charriage $n^{\circ} 1$

Concentration dans la rivière en amont de la prise d'eau : $1,087 \%$ 
4. -- Si la crue est faible, le débil linéaire au harrage peut ctre sensiblement égal ou inférieur à celui du canal d'amenée.

Le chenal principal gagne directement le caual d'amenće (photos 15 et 16) dans lequel pinetre le plus fort du courant et du débit de charriage.

Le courant se heurte aux vannes de chasse et dévic vers la chambre de prise; en profondeur, i) amorce un mouvement de retour parallèle aux vannes et dirigé vers le bas.

Ce mouvement ne suffit pas à empècher l'entrée des matériaux charriés, amenés par le chenal principal: le canal d'amenée se comble d'abord de dépôts qui, peu à peu, atteignent le niveau du seuil de l'échancrure et finalement pémétrent dans la chambre de prise (photos 15 et 16 ).

Dans le cas des rivières à matériau de fond fin, i] peut se produire des affouillements locaux en amont de la combure du senil de prise et du mur guideau; le courant hélicö̈dal caractérisant les affouillements au voisinage du seuil de prise Iransporte les malériaux vers l'intérieur du canal d'amenée, où les dépôts prennent une disposilion dissymétrique (photo 15).

Pour la valeur 0,5 du rapport entre débit linéaire au barrage et débit linéaire au canal d'amenée, le tableau II donne les valeurs $a$ ), $b$ ), c) définies au paragraphe 3 ci-dessus.

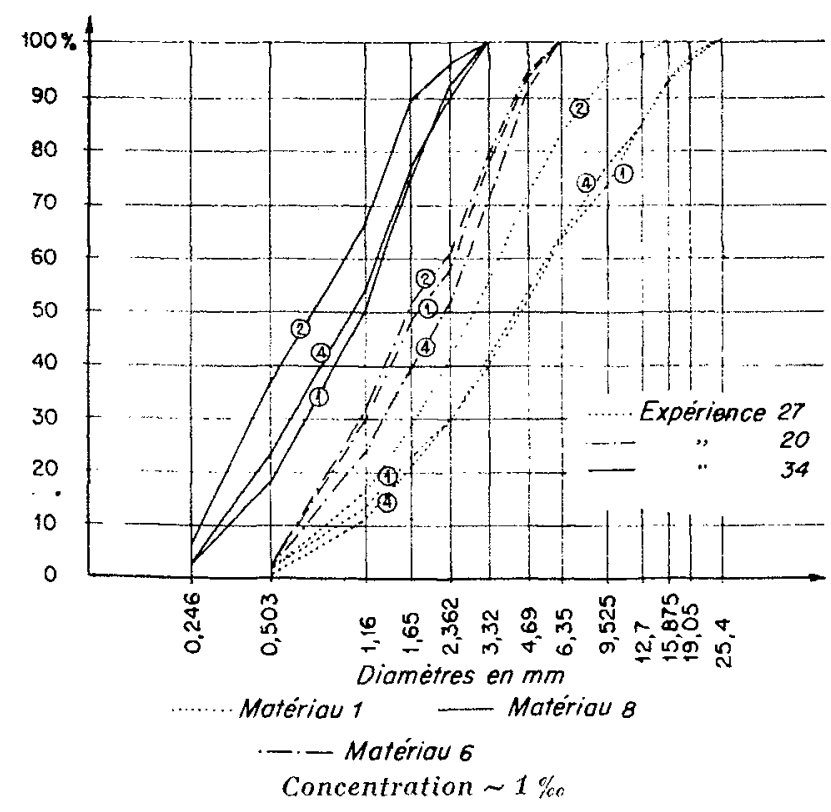

Fic, 5. - Fonctionnement anormal. Courbes granulomettiques des malériaux solides do chaviage.

(1) Rivière en amont du barrage:

(2) Rivière en aval du barrage;

(4) Prise d'eau.
La représentation graphique de ces résultats (figures 1 et 2), correspondant à des débits réparlis pour $62,8 \%$ au barrage et $37,2 \%$ à la prise, montre dans ce cas leur caractère défavorable.

La figure 5 révèle un très faible triage entre les apports de la rivière et les matériaux dérivés. Dans tous les cas, le charriage franchissant le barrage est d'une granulométrie plus fine que les apports de la rivière.

5. - Quelle que soit la répartition des débits, Ia surélévation du plan d'eau dans le canal đ'amenée provoque un petit écoulement à travers la barbacane en direction du barrage.

Près du mur guideau, côté rivière, on observe un écoulement tumultueux s'accompagnant d'affouillements localisés (photos 11 à 16).

6. - Les résultats obtenus dans les cas de fonctionnement anormal permettent de dire que ce type de prise garantit des conditions de dérivation convenables pendant un laps de temps plus grand que celui nécessaire au personne] d'exploitation pour effectuer les manœuvres voulues aux chasses et à la prise.

7. - L'ouverture des vannes de chasse place une prise d'eau de ce type dans des conditions normales de fonctionnement.

Les conditions anormales sont caractérisées par l'élévation des dépôts en amont de l'entrée du canal d'amenée et, dans quelques cas, par un certain étalement de la rivière. Par suite, l'ouverture des vannes de chasse amorce une érosion intense de ces dépôts; en peu de temps, on voit s'établir une nonvelle configuration correspondant aux conditions normales de fonctionnement telles qu'elles résultent de la répartition des débits entre barrage, chasse et prise.

Autrement dil, on passe rapidement du fonclionnement anormal au fonctionnement normal par l'intermédiaire d'une phase d'érosion brève, mais extrêmement intense; les matériaux ainsi mis en mouvement poursuivent en aval vers le canal d'amenée et les pertuis de chasse.

\section{Cas d'emploi de ce type de prise d'eau}

Les bons rẻsultats expérimentaux obtenus dans le cadre du fonctionnement normal de ce type de prise d'eau montrent que son domaine d'application aux problèmes posés par les dérivations en rivières est très large.

En effet, de bons résultats ont pu être mis en évidence dans les conditions d'essai suivantes:

a) Large variation des débits de crue;

b) Diverses rćpartitions des débits entre l'ouvrage de chasse et la prise, pour divers débits de la rivière; 
c) Diverses granulométries des matériaux de charriage;

d) Diverses concentrations des apports solides de la rivière.

Ce type de prise d'eau ne se trouve pas limité, dans les applications, par l'importance des crues de la rivière. Il ne se trouve pas limité non plus du côté des fortes pentes, car son comportement en présence d'un charriage à grosse granulométrie et à forte concentration est aussi satisfaisant que dans le cas de matériaux plus fins; à ce point de vue, ses limites sont plutòt constituées par les rivières à très faible pente dont le lit se compose de matériaux sablo-limoneux, que la turbulence de l'écoulement dans le canal d'amenée peut mettre en suspension. Son fonctionnement demeure satisfaisanl, que les concentrations de charriage soient fortes ou faibles.

Le domaine d'utilisation de ce type de prise s'étend aux torrents, aux rivières torrentielles, aux rivic̀res à pente moyenne, et, à la limite, aux rivières à lits sablo-limoneux.

Sa capacité de dérivation est illimilce. Jusqu'à $25 \mathrm{~m}^{3} / \mathrm{s}$ (et davantage si les conditions sont farorables), le dispositif de dérivation est très simple. On peut toujours dériver des débits supérieurs avec des ouvrages offrant les mêmes caractéristiques de fonctionnement, mais d'un dessin un peu différent.

Quelle que soit l'intensité de la crue, le fonctionnement normal des ouvrages assure l'évacuation du débit de charriage en aval du barrage et de l'ouvrage de chasse sous l'impulsion des excédents de débit.

Des quantités insignifiantes de limons et de sables (exceptionnellement de graviers) peuvent pénétrer dans la prise après avoir été mis en suspension par l'action des tourbillons sur les dépôts. Dans chạue cas particulier, des disposilifs simples permeltent d'ćliminer l'éventualité de l'intrusion de ces matériaux fins.
Du reste, ces matériaux charriés fins ont des dimensions semblables à celles des matériaux transportés en suspension par la rivière : ceux qui parviennent à pénétrer dans la prise poursuivent vers le dessableur.

Ce nouveau type de prise présente deux cas de fonctionnement :

1. En hautes eaux, et en exploitation normale, on joue sur louverture des vannes de chasse et de celles de la prise;

$2^{\circ}$ En étiage, les vannes de chasse sont fermées et celles de la prise suffisamment ouvertes.

Si l'arrivée d'une crue gonfle subitement de très basses eaux, la prise continue à fonctionner correctement pendant une durée supérieure au délai nécessaire à la manœuvre des vannes de chasse et de dérivation.

On passe rapidement $d u$ fonctionnement anormal au fonctionnement normal par l'intermédiaire d'une phase d'érosion brève mais intense; les matériaux charriés sont entraînés à l'aval de l'ouvrage de chasse.

Les corps flottants acheminés par la rivière peuvent être évacués à l'aval par-dessus les vannes de chasse.

Le caractère uniforme et permanent de leur modalité d'exploitation entraine pour les ouvrages des conditions de fonctionnement bien déterminces, ce qui facilite leur calcul.

La simplicité et les dimensions modestes de ce type de prise expliquent l'importance des économies initiales réalisées lors de la construction par rapport aux autres types. Pour les mêmes raisons, les frais d'entretien sont aussi faibles que possible.

Les frais d'exploitation sont réduits en raison de la facilité de manœuvre des ouvrages et du petit nombre d'organes mobiles. 